\title{
New effective moduli of uniqueness and uniform a-priori estimates for constants of strong unicity by logical analysis of known proofs in best approximation theory
}

\author{
Ulrich Kohlenbach \\ Fachbereich Mathematik, J.W. Goethe-Universität \\ Robert-Mayer-Str. 6-10, 60054 Frankfurt am Main, FRG
}

\begin{abstract}
Let $U$ and $V$ be complete separable metric spaces, $V_{u}$ compact in $V$ and $G: U \times V \rightarrow \mathbb{R}$ a continuous function. For a large class of (usually non-constructive) proofs of uniqueness theorems

$$
\bigwedge u \in U, v_{1}, v_{2} \in V_{u}\left(G\left(u, v_{1}\right)=\inf _{v \in V_{u}} G(u, v)=G\left(u, v_{2}\right) \rightarrow v_{1}=v_{2}\right)
$$

one can extract an effective modulus of uniqueness $\Phi$ by logical analysis, i.e.$$
\bigwedge u \in U, v_{1}, v_{2} \in V_{u}, n \in \mathbb{N}\left(\bigwedge_{i=1}^{2}\left(G\left(u, v_{i}\right)-\inf _{v \in V_{u}} G(u, v) \leq 2^{-\Phi u n}\right) \rightarrow d_{V}\left(v_{1}, v_{2}\right) \leq 2^{-n}\right) .
$$

Since $\Phi$ does not depend on $v_{1}, v_{2}$ it is an a-priori estimate, which generalizes the notion of strong unicity in Chebycheff approximation theory. This applies to uniqueness proofs in Chebycheff approximation, $\|\cdot\|_{1}$-approximation of $f \in C[0,1]$ and best uniform approximation by polynomials having bounded coefficients. Here we continue our proof-theoretic analysis started in [21]. A simplification of a proof by Young/Rice and a variant due to Borel are analysed yielding explicit moduli $\Phi$ and uniform a-priori (lower) estimates for strong unicity which are significantly better than those obtained from de La Vallée Poussin's uniqueness proof in [21]. It is explained how the numerical content of the three proofs depends on the logical form whereby certain analytical lemmas (e.g. the alternation theorem) play a key-role. The numerical results obtained from Young/Rice's proof are best and substantially improve known estimates obtained by D. Bridges by an n (=dimension of the Haar space) in the exponent.
\end{abstract}

\section{Introduction}

In this paper we are concerned with the extraction of effective a-priori estimates, called moduli of uniqueness, from ineffective proofs of uniqueness of best approximations. Roughly speaking, a modulus of uniqueness is a quantitative version of an uniqueness theorem.

Let us consider the following situation:

Suppose that $U$ and $V$ are complete separable metric spaces (abbreviation: CSM-spaces) and that $V_{u}$ is a compact subset of $V$ for each $u \in U$. Furthermore let $G: U \times V \rightarrow \mathbb{R}$ be a continuous function. Assume now that $G(u, \cdot)$ has at most one zero in $V_{u}$, i.e.

$$
\text { (1) } \bigwedge u \in U, v_{1}, v_{2} \in V_{u}\left(G\left(u, v_{1}\right)=0=G\left(u, v_{2}\right) \rightarrow v_{1}=v_{2}\right) .
$$

By a modulus of uniqueness for this situation we mean an operation $\Phi$ such that $\Phi u: \mathbb{Q}_{+}^{*} \rightarrow \mathbb{Q}_{+}^{*}$ and

$$
\text { (2) } \bigwedge_{u} \in U, v_{1}, v_{2} \in V_{u}, q \in \mathbb{Q}_{+}^{*}\left(\bigwedge_{i=1}^{2}\left(\left|G\left(u, v_{i}\right)\right| \leq \Phi u q\right) \rightarrow d_{V}\left(v_{1}, v_{2}\right) \leq q\right) \text {. }
$$


Such a modulus can be used for a computation with prescribed precision of the uniquely determined zero of $G(u, \cdot)$ in $V_{u}$ (if such a zero exists). Take an algorithm $\Psi$ which computes a $2^{-k}$-zero of $G(u, \cdot)$, i.e.

(3) $\bigwedge u \in U, k \in \mathbb{N}\left(|G(u, \Psi u k)| \leq 2^{-k} \wedge \Psi u k \in V_{u}\right)$.

Then this algorithm when started with $u$ and $k:=\Phi u\left(2^{-n}\right)$ will compute the uniquely determined zero with an error $\leq 2^{-n}$.

In the case of best approximation theory the concept of a modulus of uniqueness generalizes the notion of (a constant of) strong unicity (which is important for Chebycheff approximation) and immediately gives a modulus of pointwise continuity for the corresponding projection operator (see Prop. 2.4 below).

In [21] we used a proof-theoretic method from [23] (more precisely, a new combination of Gödel's so-called functional interpretation with a recursion-theoretic majorization technique) to establish a general meta-theorem which guarantees the extractability of effective moduli of uniqueness for a large class of (ineffective) uniqueness proofs. Applied to the uniqueness proof for best Chebycheff approximation of $f \in C[0,1]$ by algebraic polynomials of degree $\leq n$ by [28] we obtained a concrete modulus of uniqueness and an effective (a-priori lower estimate of a) constant of strong unicity as well as a new quantitative version of the well-known alternation theorem.

In this paper we first present the main results of [21] in usual mathematical terms avoiding as much as possible technical notions from mathematical logic. Then we analyse a further proof of uniqueness for best Chebycheff approximation (also for general Haar spaces), which is a simplification of the proof from [32] and [29], and discuss a variant of this proof for the polynomial case only due to Borel [4]. The extraction of moduli of uniqueness and constants of strong unicity from these proofs is much easier compared to de La Vallée Poussin's proof. The best results (also for the polynomial case) follow from our simplified version of the proof by [32] and [29]. We use these estimates to improve results by D.S. Bridges in [6],[7] and [8], who first obtained effective a-priori estimates for these data in Chebycheff approximation theory (working entirely within the framework of Bishop's constructive analysis, see [1]), by an $n$ (=dimension of the Haar space) in the exponent. By our meta-theorem mentioned above it follows that our results are provable within intuitionistic arithmetic and hence also in constructive mathematics in the sense of Bishop. However such a (in general quite complicated) constructive verification of the estimates is only of foundational interest and does not contribute to the numerical results which are obtained by following the logical structure and the data of the classical (ineffective) proof. It is the logical form in which certain lemmas (e.g. the alternation theorem) are used in these uniqueness proofs which is crucial for the numerical content.

\section{General results}

In order to formulate our general theorem on the existence of effective moduli of uniqueness we have to introduce some notions from mathematical logic and constructive analysis which we present here as informal as possible (for details see [21]).

First we sketch how elements of a CSM-space $(X, d)$ can be represented by sequences $f: \mathbb{N} \rightarrow \mathbb{N}$ of natural numbers such that

1) each $x \in X$ is represented by some (not uniquely determined) $f \in \mathbb{N}^{\mathbb{N}}$, 
2) each $f \in \mathbb{N}^{\mathbb{N}}$ represents a unique element $x_{f} \in X$,

3) the equivalence relation $f_{1}={ }_{X} f_{2}: \equiv\left(f_{1}\right.$ and $f_{2}$ represent the same element in $\left.X\right)$, i.e. $d\left(x_{f_{1}}, x_{f_{2}}\right)=0$, has the logical form $\bigwedge_{k} \in \mathbb{N} A_{0}\left(f_{1}, f_{2}, k\right)$ where $A_{0}$ is quantifier-free.

We indicate this representation for the special space $X=\mathbb{R}$ :

Rational numbers can easily be coded as pairs of natural numbers so that one can define primitive recursive operations $+_{\mathbb{Q}}, \cdot_{\mathbb{Q}},|\cdot|_{\mathbb{Q}}$ and predicates $<_{\mathbb{Q}}, \leq_{\mathbb{Q}}$. Real numbers are represented as sequences of rational numbers and thus, under the coding of rational numbers by natural numbers, as functions $f: \mathbb{N} \rightarrow \mathbb{N}$ such that

$$
\text { (4) } \bigwedge_{n \in \mathbb{N}}\left(\left|f(n)-_{\mathbb{Q}} f(n+1)\right|_{\mathbb{Q}}<_{\mathbb{Q}}\left\langle 2^{-n-1}\right\rangle\right),
$$

where $\langle q\rangle$ denotes a natural number which codes the rational number $q$.

(4) implies

$$
\begin{aligned}
& \bigwedge_{n} \in \mathbb{N} \bigwedge_{k, m ; k}>m \geq n\left(|f(m)-\mathbb{Q} f(k)|_{\mathbb{Q}} \leq_{\mathbb{Q}} \sum_{i=m}^{k-1}\left|f(i)-_{\mathbb{Q}} f(i+1)\right|_{\mathbb{Q}}\right. \\
& \left.\leq_{\mathbb{Q}} \sum_{i=n}^{\infty}|f(i)-\mathbb{Q} f(i+1)|_{\mathbb{Q}}<_{\mathbb{Q}}\left\langle 2^{-n}\right\rangle\right) .
\end{aligned}
$$

Therefore each $f \in \mathbb{N} \rightarrow \mathbb{N}$ which satisfies (4) represents a Cauchy sequence of rational numbers with Cauchy modulus $2^{-n}$. In the other direction for each Cauchy sequence $f$ with modulus $2^{-n}$, $f^{\prime}(n):=f(n+1)$ satisfies (4). In order to achieve that every function $f \in \mathbb{N} \rightarrow \mathbb{N}$ represents a real number, we introduce the following primitive recursive construction:

$$
\text { (5) } \widehat{f}(n):=\left\{\begin{array}{l}
f(n), \text { if } \bigwedge_{k}<n\left(|f(k)-\mathbb{Q} f(k+1)|_{\mathbb{Q}}<_{\mathbb{Q}}\left\langle 2^{-k-1}\right\rangle\right), \\
f(k) \text { for the least } k<n \text { with }\left|f(k)-_{\mathbb{Q}} f(k+1)\right|_{\mathbb{Q}} \geq_{\mathbb{Q}}\left\langle 2^{-k-1}\right\rangle, \text { else. }
\end{array}\right.
$$

For all $f \in \mathbb{N} \rightarrow \mathbb{N}, \widehat{f}$ satisfies (4). If (4) is already fulfiled by $f$ then does not change $f: \bigwedge_{n} \in$ $\mathbb{N}(f(n)=\widehat{f}(n))$. Thus each function $f$ codes a uniquely determined real number, namely the real number which is given by the Cauchy sequence coded by $\widehat{f}$.

Using this construction one can reduce quantification over $\mathbb{R}$ to quantification over $\mathbb{N}^{\mathbb{N}}$. On the codes of real numbers (i.e. on $\mathbb{N}^{\mathbb{N}}$ ) $f_{1}, f_{2}$ we define an equivalence relation $=\mathbb{R}$ by

$$
\text { (6) } f_{1}={ }_{\mathbb{R}} f_{2}: \equiv \bigwedge_{n}\left(\left|\widehat{f}_{1}(n+1)-_{\mathbb{Q}} \widehat{f}_{2}(n+1)\right|_{\mathbb{Q}}<_{\mathbb{Q}}\left\langle 2^{-n}\right\rangle\right) \text {. }
$$

One easily verifies (using that $\widehat{f}_{1}$ and $\widehat{f}_{2}$ satisfy (4)) that $f_{1}={ }_{\mathbb{R}} f_{2}$ holds if and only if $f_{1}$ and $f_{2}$ represent the same real number.

(7) $f_{1}<_{\mathbb{R}} f_{2}: \equiv \bigvee_{n}\left(\widehat{f}_{2}(n+1)-\mathbb{Q} \widehat{f}_{1}(n+1) \geq_{\mathbb{Q}}\left\langle 2^{-n}\right\rangle\right), \quad f_{1} \leq_{\mathbb{R}} f_{2}: \equiv \neg\left(f_{2}<_{\mathbb{R}} f_{1}\right)$.

$f_{1}={ }_{\mathbb{R}} f_{2}$ and $f_{1} \leq_{\mathbb{R}} f_{2}$ are purely universal formulas, i.e. formulas having the form

$\bigwedge_{n} \in \mathbb{N} A_{0}\left(f_{1}, f_{2}, n\right)$ where $A_{0}$ is quantifier-free, whereas $f_{1}<_{\mathbb{R}} f_{2}$ is an existential formula $\bigvee_{n} \in$ $\mathbb{N} A_{0}\left(f_{1}, f_{2}, n\right)$. Using this representation of $\mathbb{R}$ one can represent arbitrary CSM-spaces $(X, d)$ as spaces $\left(\mathbb{N}^{\mathbb{N}}, d_{X}\right)$, where $d_{X}$ represents a pseudo-metric on $\mathbb{N}^{\mathbb{N}}$ such that the set of equivalence classes of $\mathbb{N}^{\mathbb{N}}$ w.r.t. $f_{1}={ }_{X} \quad f_{2}: \equiv\left(d_{X}\left(f_{1}, f_{2}\right)==_{\mathbb{R}} 0\right)$ is isometric isomorphic (w.r.t. the metric induced by $\left.d_{X}\right)$ to $(X, d)$ (for details see [21]). For e.g. $\left(C[0,1],\|\cdot\|_{\infty}\right)$ (where $\|\cdot\|_{\infty}$ denotes the the 
sup norm) this means in practise that the elements $f \in C[0,1]$ are always endowed with a modulus $\omega_{f}: \mathbb{Q}_{+}^{*} \rightarrow \mathbb{Q}_{+}^{*}$ of uniform continuity on $[0,1]$, i.e.

$$
\text { (8) } \bigwedge x, y \in[0,1], q \in \mathbb{Q}_{+}^{*}\left(|x-y|<\omega_{f}(q) \rightarrow|f(x)-f(y)|<q\right) .
$$

This notion of a modulus of continuity is common in logic and constructive analysis but it differs from the usual notion in numerical analysis.

Functions $F: X \rightarrow Y$ for CSM-spaces $X, Y$ are now given as functions $\Phi_{F}: \mathbb{N}^{\mathbb{N}} \rightarrow \mathbb{N}^{\mathbb{N}}$ which are extensional w.r.t. $=_{X},=_{Y}$, i.e. $f_{1}={ }_{X} f_{2} \rightarrow \Phi_{F}\left(f_{1}\right)=_{Y} \Phi_{F}\left(f_{2}\right)$. Thus we have to deal not only with functions $f: \mathbb{N} \rightarrow \mathbb{N}$ but also with functions which map functions to functions and so on. Hence in order to formalize proofs in analysis we use a system $\mathcal{A}$ which is formulated in the language of function(al)s of finite type:

Finite types are inductively defined as follows:

(i) 0 is a type, (ii) if $\rho$ and $\tau$ are types then $\rho(\tau)$ is also a type.

0 denotes the type of natural numbers, i.e. terms denoting natural numbers have type $0 . \rho(\tau)$ is the type of functions which map type- $\tau$-objects to type- $\rho$-objects, so e.g. $f: \mathbb{N}^{\mathbb{N}} \rightarrow \mathbb{N}^{\mathbb{N}}$ has type $0(0)(0(0))$. Functions having types which are more complex then $0(0)$ are usually called functionals. $\mathcal{A}$ denotes the extension of the usual arithmetic to the language with variables for all types plus the following schema of recursive search

$$
A C^{0,0}{ }_{\text {qf }}: \bigwedge^{0} \bigvee^{0} A_{0}(x, y) \rightarrow \bigvee_{f^{0(0)}} \wedge^{0} A_{0}(x, f(x)),
$$

where $A_{0}$ is a quantifier-free formula.

Furthermore one can define in $\mathcal{A}$ terms by primitive recursion. The functionals which are definable in this way are the so-called primitive recursive functionals of [15] and [13]. For an exact definition of $\mathcal{A}$ we refer to [21], where $\mathcal{A}$ is denoted by $E-P A^{\omega}+A C^{0,0}$-qf and the calculus of primitive recursive functionals by $T$. If we omit $A C^{0,0}$-qf and allow only constructive logic we obtain a system of intuitionistic arithmetic which we call $\mathcal{A}^{i}$ (this is $W E-H A^{\omega}$ from [21]). Every proof in $\mathcal{A}^{i}$ is in particular a constructive proof in the sense of Bishop's constructive analysis [1] (see [2] where $\mathcal{A}^{i}$ is used for formalizing constructive mathematics).

There are primitive recursive functionals of type $0(0)$ (i.e. functions) which are not primitive recursive in the usual sense of ordinary recursion theory (as developed e.g. in [16]). For example the Ackermann function is a primitive recursive functional of $\mathcal{A}$. This is due to the fact that in $\mathcal{A}$ primitive recursion in higher types is allowed. If the schema of induction in $\mathcal{A}$ is restricted to formulas of the form $\bigvee_{x^{0}} A_{0}(x)$ where $A_{0}$ is quantifier-free and only primitive recursion in type 0 is allowed (but with higher type objects as parameters possibly) then the definable functions of this restricted system $\widehat{\mathcal{A}} \uparrow$ (called $E \widehat{-P} A^{\omega} \uparrow+A C^{0,0}-$ ff in [21]) are just the usual primitive recursive ones. All of our results in the following hold for both $\mathcal{A}$ and $\widehat{\mathcal{A}} \uparrow$ with the corresponding notion of primitive recursive functionals (Similar for the notions "constructively definable" CSM-space, function etc. below). For notational simplicity we formulate them only for the case $\mathcal{A}$.

In applications to concrete mathematical proofs usually only very special instances of induction occur and thus the functionals extracted from these proofs are simple mathematical operations in the data of the proof (instead of being merely primitive recursive).

A CSM-space $(X, d)$ represented as $\left(\mathbb{N}^{\mathbb{N}}, d_{X}\right)$ is constructively definable if $d_{X}$ is a primitive recursive functional which represents provable in $\mathcal{A}$ a pseudo-metric on $\mathbb{N}^{\mathbb{N}}$. Examples are 
$\left(C[0,1],\|\cdot\|_{\infty}\right),\left(\mathbb{R}^{n},\|\cdot\|_{E}\right)$ and $\left(L_{p},\|\cdot\|_{p}\right)(1 \leq p<\infty)$.

A constructive function $F: X \rightarrow Y$ is given by a primitive recursive functional $\Phi_{F}^{0(0)(0(0))}$ on the representatives which is (provable in $\mathcal{A}$ ) extensional w.r.t. $={ }_{X},=_{Y}$.

Many proofs in analysis use besides arithmetical tools available in $\mathcal{A}$ only analytical lemmas having the form

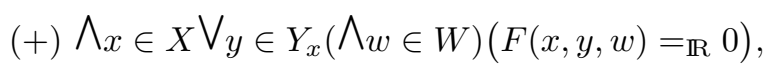

where $X, Y, W$ are constructively definable CSM-spaces, $Y_{x}$ is compact in $Y$ and $F$ is a constructive function.

Examples of sentences $(+)$ are

1) The intermediate value theorem for $f \in C[0,1]$;

2) The attainment of the maximum $\wedge f \in C[0,1] \bigvee_{x_{0}} \in[0,1]\left(f\left(x_{0}\right)=\sup _{x \in[0,1]} f(x)\right)$;

3) The existence theorem of Cauchy-Peano on the solvability of ordinary differential equations.

Such lemmas $(+)$ simply can be used as implicative assumptions in the course of the logical extraction of the data. It then turns out that proofs of such lemmas are not relevant for the numerical data as far as conclusions of the form $\bigwedge_{x} \in X \bigvee_{n} \in \mathbb{N} A_{0}(x, n)$ are considered (Examples 2) and 3) are unprovable in $\mathcal{A}$ while 1) is provable in $\mathcal{A}$ but not in $\mathcal{A}^{i}$ ).

In the following let $X, Y, U, V, W$ be constructively definable CSM-spaces, $F: U \times X \times Y \rightarrow \mathbb{R}$ and $G: U \times V \times W \rightarrow \mathbb{R}$ constructive (and therefore continuous) functions. Furthermore let $\left(Y_{x}\right)_{x \in X},\left(V_{u}\right)_{u \in U}$ be constructive $X, U$-families of compact sets in $Y, V$.

Theorem 2.1 ([21]) Suppose that $A_{1}\left(u^{0(0)}, v^{0(0)}, k^{0}, m^{0}\right)$ in the language of $\mathcal{A}$ is a formula having the logical form $\bigvee_{\underline{l}} A_{0}$ where $\underline{\underline{l}}$ is a tuple of type-0/0(0)-variables, $A_{0}$ is quantifier-free and $A_{1}$ has only the free variables $u, v, k, m$. Furthermore, assume that $A_{1}$ is extensional w.r.t. $=_{U},=_{V}$ in $u, v$ (provable in $\mathcal{A}$ ) so that $A_{1}$ is really a statement about $U, V$-objects.

Then the following rule holds

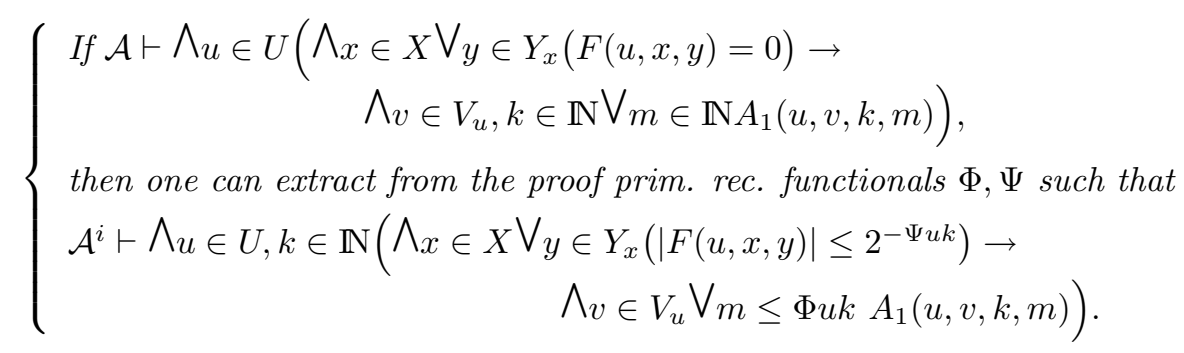

(An analogous result holds for $\widehat{\mathcal{A}} \uparrow$ ).

Remark: The assumption $\mathcal{A} \vdash \bigwedge u \in U(\ldots)$ in 2.1 and in the following means that this proposition is provable in $\mathcal{A}$ for the representation of $U, X$ etc. as sketched above. The functionals $\Phi, \Psi$ operate on the representatives of the elements of $U$ and are in general not extensional with respect to $u_{1}=_{U} u_{2}\left(\equiv d_{U}\left(u_{1}, u_{2}\right)=0\right)$. For $C[0,1],\|\cdot\|_{\infty}$ this means that $\Phi$ uses $f$ endowed with a modulus of uniform continuity on $[0,1]$. On these enriched data, $\Phi$ is extensional in our applications. 
We now apply 2.1 to uniqueness theorems:

\section{Theorem $2.2([21])$}

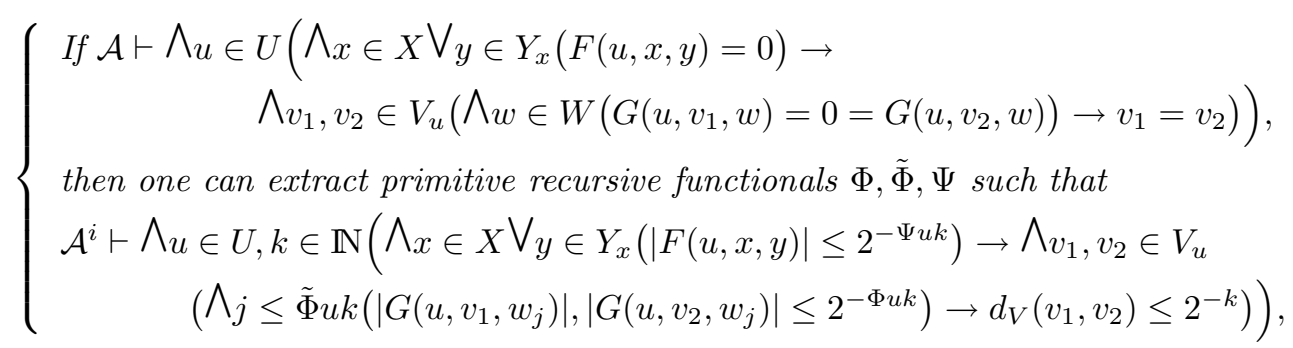

where $\left(w_{n}\right)_{n \in \mathbb{N}}$ is dense in $W$.

2.2 can be applied to uniqueness theorems in best approximation theory. Here one often is concerned with the computation of a uniquely determined minimal point of a function $G(u, \cdot): V \rightarrow \mathbb{R}$ on a compact set $V_{u} \subset V$ for $u \in U$. If $G$ is constructive then $\tilde{G}(u, v):=G(u, v)-\inf _{\tilde{v} \in V_{u}} G(u, \tilde{v})$ is also constructively definable. For all $u \in U, v \in V_{u}$ one has $\tilde{G}(u, v)=0 \leftrightarrow G(u, v)=\inf _{\tilde{v} \in V_{u}} G(u, \tilde{v})$. Thus under the assumptions of theorem 2.2 (and $\bigwedge_{x} \in X, m \in \mathbb{N} \bigvee y \in Y_{x}\left(|F(u, x, y)| \leq 2^{-m}\right)$ ) one can extract a modulus $\Phi$ such that

$$
\bigwedge u \in U, k \in \mathbb{N}, v_{1}, v_{2} \in V_{u}\left(G\left(u, v_{1 / 2}\right)-\inf _{v \in V_{u}} G(u, v) \leq 2^{-\Phi u k} \rightarrow d_{V}\left(v_{1}, v_{2}\right) \leq 2^{-k}\right) .
$$

In the introduction we already indicated how such a modulus of uniqueness $\Phi$ can be used to compute with prescribed precision the unique minimal point $v_{u}$ under the assumption of its existence. Of course ineffectively such a point always exists since the continuous function $G(u, \cdot)$ assumes its infimum on the compact set $V_{u}$. However the fact that a continuous function assumes its infimum on compact sets is not provable in $\mathcal{A}$. Nevertheless, by an $\varepsilon$-refinement of the argument given in the introduction one can derive the computability of $v_{u}$ even in $\mathcal{A}^{i}$ :

Theorem 2.3 ([21])

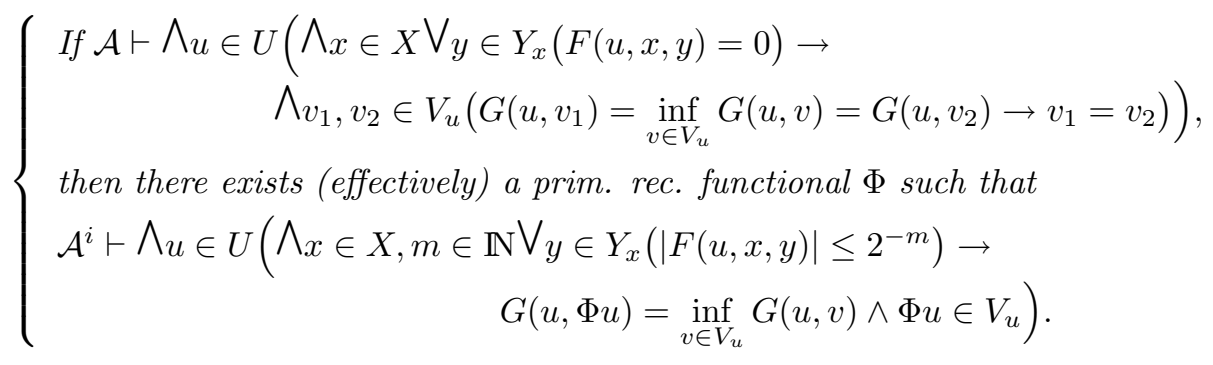

By the uniqueness of the minimal point, $\Phi$ is extensional w.r.t. $={ }_{U}$ and therefore computes a function $U \rightarrow V$.

In our examples, the analytical tools $\bigwedge_{x} \in X \bigvee y \in Y_{x}(F=0)$ used in proving the uniqueness theorems are in general not provable in $\mathcal{A}$ (and therefore a-fortiori not in $\mathcal{A}^{i}$ ) however their $\varepsilon^{-}$ weakenings $\bigwedge_{x} \in X, m \in \mathbb{N} \bigvee y \in Y_{x}\left(|F(u, x, y)| \leq 2^{-m}\right)$ are even $\mathcal{A}^{i}$-provable. Hence 2.3 yields

$$
\mathcal{A}^{i} \vdash \bigwedge_{u} \in U\left(G(u, \Phi u)=\inf _{v \in V_{u}} G(u, v) \wedge \Phi u \in V_{u}\right)
$$


and so the existence of a $v_{u} \in V_{u}$ such that $G\left(u, v_{u}\right)=\inf _{v \in V_{u}} G(u, v)$ is provable in every framework of constructive analysis (as intuitionistic analysis or Bishop's constructive analysis or recursive analysis).

Let $(X,\|\cdot\|)$ be a real normed space and $E \subset X$ a finite dimensional subspace of $X$. It is well-

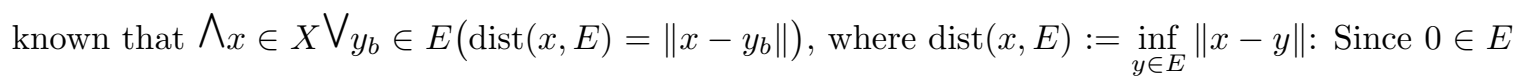
it follows that $\operatorname{dist}(x, E) \leq\|x\|$ and therefore $\left\|y_{b}\right\| \leq 2\|x\|$. Hence $\operatorname{dist}(x, E)=\operatorname{dist}\left(x, K_{x}\right)$, where $K_{x}:=\{y \in E:\|y\| \leq 2\|x\|\}$ is compact. The existence of a best approximation $y_{b}$ now follows from the fact that the continuous function $\Phi_{x}(y):=\|x-y\|$ assumes its minimum on compact sets.

In many important cases the spaces $X, E$ and $K_{x}$ are constructively definable and the uniqueness of the best approximation of $x \in X$ in $E$ is proved in $\mathcal{A}$ plus lemmas having the form $\bigwedge_{u} \in U \bigvee_{v} \in V_{u}\left(F(u, v)=\mathbb{R}_{0} 0\right)$; see the examples 2.5 below. Thus theorem 2.2 allows the construction of moduli of uniqueness $\Phi$ in these cases. $\Phi$ can be easily extended to $q \in \mathbb{Q}_{+}^{*}$ (instead of $2^{-n}$ ) and to a modulus of uniqueness $\widehat{\Phi}$ on $E$ instead of $K_{x}$ (although $E$ is not compact in $X$ ) (see $[21])$, i.e. $\widehat{\Phi}: \mathbb{Q}_{+}^{*} \rightarrow \mathbb{Q}_{+}^{*}$ such that

$$
\bigwedge_{x} \in X, y_{1}, y_{2} \in E, q \in \mathbb{Q}_{+}^{*}\left(\bigwedge_{i=1}^{2}\left(\left\|y_{i}-x\right\|-\operatorname{dist}(x, E) \leq \widehat{\Phi} x q\right) \rightarrow\left\|y_{1}-y_{2}\right\| \leq q\right) .
$$

The next result shows that a modulus of uniqueness is a generalization of a constant of strong unicity (in the sense of [27],[10]) and immediately yields a modulus of pointwise continuity for the projection $X \rightarrow E$ :

Proposition 2.4 ([21]) Let $\Phi$ be a modulus of uniqueness for the best approximation of $x \in X$ in $E$ (The special case where $y_{2}$ is taken to be the best approximation suffices). Then the following holds:

1) $\frac{1}{2} \Phi$ is a modulus of pointwise continuity for the projection $\mathcal{P}: X \rightarrow E$ which maps $x \in X$ to its best approximation $y_{b}$ in $E$, i.e.

$$
\bigwedge x, x_{0} \in X, q \in \mathbb{Q}_{+}^{*}\left(\left\|x-x_{0}\right\| \leq \frac{1}{2} \Phi x_{0} q \rightarrow\left\|\mathcal{P}(x)-\mathcal{P}\left(x_{0}\right)\right\| \leq q\right) .
$$

If $\Phi$ is linear in $q$, i.e. $\Phi x q=q \cdot \gamma(x)$, then

2) $\gamma(x)$ is a (lower estimate of the) constant of strong unicity, i.e.

$$
\bigwedge x \in X, y \in E\left(\|x-y\| \geq\left\|x-y_{b}\right\|+\gamma(x) \cdot\left\|y-y_{b}\right\|\right),
$$

where $y_{b}$ is the best approximation of $x$,

3) $\lambda(x):=\frac{2}{\gamma(x)}$ is a (pointwise) Lipschitz constant of $\mathcal{P}$, i.e.

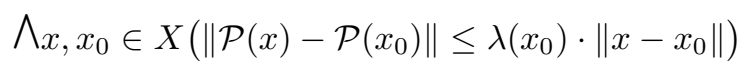

(3) follows immediately from 2) by the proof of a lemma from [10], p.82, formulated there for Chebycheff approximation). 
Example 2.5 In the following examples there are well-known proofs for the uniqueness of best approximations which can be carried out in $\mathcal{A}+(A)$, where $(A)$ is the sentence $\bigwedge_{f} \in C[0,1] \bigvee_{x_{0}} \in$ $[0,1]\left(f\left(x_{0}\right)=\sup _{x \in[0,1]} f(x)\right)$. Because of its logical form, $(A)$ is an admissible analytical tool for our proof analysis and thus 2.2 and 2.3 are applicable:

1) Let $\left(\Phi_{1}, \ldots, \Phi_{n}\right)$ be an Chebycheff system in $[0,1]$. Then (due to [9],[18])

$$
\bigwedge f \in C[0,1] \bigvee ! \phi \in \operatorname{Lin}_{\mathbb{R}}\left(\Phi_{1}, \ldots, \Phi_{n}\right)\left(\|f-\phi\|_{\infty}=\operatorname{dist}\left(f, \operatorname{Lin}_{\mathbb{R}}\left(\Phi_{1}, \ldots, \Phi_{n}\right)\right) .\right.
$$

2) Best $\|\cdot\|_{1}$-approximation of continuous functions: $\|f\|_{1}:=\int_{0}^{1}|f(x)| d x$ defines a norm on $C[0,1]$.

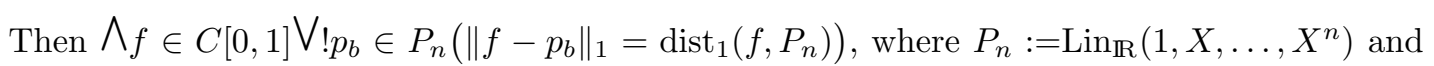
$\operatorname{dist}_{1}\left(f, P_{n}\right):=\inf _{p \in P_{n}}\|f-p\|_{1}$. This also generalizes to arbitrary Chebycheff systems instead of $P_{n}$. The first uniqueness proof was given by Jackson [17]. Since this proof uses concepts from measure theory it is not clear whether it can be formalized in $\mathcal{A}+(A)$ or not. However there is a different uniqueness proof by [10] which can easily be carried out in $\mathcal{A}+(A)$. Since $\left(C[0,1],\|\cdot\|_{1}\right)$ is not complete, we have to represent this space as $\left(C[0,1],\|\cdot\|_{\infty}\right)$ i.e. the modulus of uniqueness and the algorithm are only effective in $f$ together with a modulus of uniform continuity of $f$ (instead of a -weaker- modulus of integration).

3) Let $(X,\|\cdot\|)$ be a strictly convex space. Then the following result (due to Krein) holds: Suppose that $E \subset X$ is finite dimensional, then

$\bigwedge_{x} \in X \bigvee ! y_{b} \in E\left(\left\|x-y_{b}\right\|=\operatorname{dist}(x, E)\right)$. Examples of constructively definable strictly convex spaces are $\left(L_{p},\|\cdot\|_{p}\right)$ for $1<p<\infty$. Although the uniqueness proof in this case is quite simple (see e.g. [10] ) it nevertheless yields a modulus of uniqueness which is not obvious, e.g. for the special case $L_{2}$ one obtains (for $q \leq 1$ )

$$
\Phi x q:=\min \left(1, \frac{q}{4}, \max \left(l_{x}, \frac{q}{4}\right) \cdot \frac{\gamma\left(\frac{q}{k_{x}}\right)}{1-\gamma\left(\frac{q}{k_{x}}\right)}\right),
$$

where $0<l_{x} \leq \operatorname{dist}(x, E), k_{x} \geq \operatorname{dist}(x, E)+1$ (e.g. $\left.k_{x}:=2\|x\|+1\right)$ and $\gamma(q):=q^{2} / 8$ (for details see [22]). This modulus holds for arbitrary convex subsets $E$ of $L_{2}$.

4) Chebycheff approximation by polynomials having bounded coefficients: For $0 \leq i \leq p$ let $k_{i} \in \mathbb{N}$ be such that $0<k_{0}<k_{1}<\ldots<k_{p} \leq n-1(p \leq n)$ and $a_{1}, \ldots, a_{p}, b_{1}, \ldots, b_{p} \in \overline{\mathbb{R}}:=$ $\mathbb{R} \cup\{-\infty, \infty\}$ such that

i) $a_{i} \neq+\infty$, ii) $b_{i} \neq-\infty$, iii) $a_{i} \leq b_{i}$ for $i=0,1, \ldots, p$.

$K_{n}:=\left\{\sum_{i=0}^{n} c_{i} x^{i}: a_{i} \leq c_{k_{i}} \leq b_{i} ; 0,1, \ldots, p\right\} . K_{n}$ is a convex subset of $P_{n}$. In [30] it is proved that $\bigwedge f \in C[0,1] \bigvee ! p_{b} \in K_{n}\left(\left\|f-p_{b}\right\|_{\infty}=\operatorname{dist}\left(f, K_{n}\right)\right) . K_{n}$ can be replaced by a compact subset: Let $p_{0} \in K_{n}$ and $\left\|f-p_{b}\right\|_{\infty}=\operatorname{dist}\left(f, K_{n}\right)$. Then $\left\|p_{b}\right\|_{\infty} \leq 2\|f\|_{\infty}+\left\|p_{0}\right\|_{\infty}$. Hence $\operatorname{dist}\left(f, K_{n}\right)=\operatorname{dist}\left(f, \widehat{K}_{n}\right)$, where $\widehat{K}_{n}:=\left\{p \in K_{n}:\|p\|_{\infty} \leq 2\|f\|_{\infty}+\left\|p_{0}\right\|_{\infty}\right\}$ is compact. 
Since the uniqueness proofs refered to in 1)-4) are formalizable in $\mathcal{A}+(A)$ we obtain by $2.2,2.3$ (In 1) and 2) for constructively definable Chebycheff systems in the sense of 3.2 below):

Corollary 2.6 One can construct primitive recursive (in the sense of [13]) moduli of uniqueness and primitive recursive algorithms for the best approximation in 2.5.1-2.5.4 which can be verified within $\mathcal{A}^{i}$. In particular the existence of best approximations is provable in $\mathcal{A}^{i}$ and hence in Bishop's constructive analysis (For 1) and 3) (for uniformly convex spaces) the later is due to [5],[6]; for 2) and 4) this is new).

In the following we continue our unwinding of uniqueness proofs for 2.5.1 from [21]:

In [21] we gave a proof-theoretic analysis of the most common proof for the uniqueness of best Chebycheff approximation by polynomials from [28] (as presented with all details in [26]). This proof proceeds roughly as follows:

If $p_{1}, p_{2} \in P_{n}$ are best approximations of $f$, then also $\frac{p_{1}+p_{2}}{2}$ is a best approximation of $f$ and therefore possesses an extremal alternant $x_{1}<\ldots<x_{n+2}$ of $n+2$-points in $[0,1]$ by the well-known alternation theorem. One shows that $p_{1}\left(x_{i}\right)=p_{2}\left(x_{i}\right)$ for $i=1, \ldots, n+2$ and thus $p_{1} \equiv p_{2}$. Besides purely arithmetical reasoning the sentence $(A)$ is used essentially to establish the alternation theorem (which implies $(A)$ relative to $\mathcal{A}^{i}$ already for $n=1$; see [8]).

Theorem 2.7 ([21]) Proof-theoretic analysis of the uniqueness proof from de La Vallée Poussin yields the following numerical a-priori estimates:

Let $\omega_{f}$ be modulus of continuity of $f,\|f\|_{\infty} \leq M_{f} \in \mathbb{Q}_{+}^{*}$ and define

$$
\omega_{n, M_{f}}(q):=\frac{q}{5 n^{2} \cdot\left\|M_{f}\right\|_{\infty}}, \tilde{\omega}_{f, n}(q):=\left\{\begin{array}{l}
\min \left(\omega_{n, M_{f}}\left(\frac{q}{2}\right), \omega_{f}\left(\frac{q}{2}\right)\right) \text { for } n>1 \\
1 \text { for } n=0
\end{array}\right.
$$

and for $l \in \mathbb{Q}_{+}^{*}$ :

$$
\Phi f n l:=\frac{1}{10(n+1)} \prod_{i=1}^{\left\lfloor\frac{n \dot{m}}{2}\right\rfloor}\left(2 i-\frac{1}{2}\right) \cdot \prod_{i=1}^{\left\lceil\frac{n \dot{\dot{m}}}{2}\right\rceil}\left(2 i-\frac{3}{2}\right) \cdot\left\lfloor\frac{n}{2}\right\rfloor !\left\lceil\frac{n}{2}\right\rceil ! \cdot \tilde{\omega}_{f, n}\left(\frac{l}{2}\right)^{n \dot{-1}} \cdot \tilde{\omega}_{f, n}\left(\frac{3 l}{2}\right)^{n} .
$$

Then

1) $(\Phi f n l) \cdot q$ is a linear modulus of uniqueness for $f, n$ such that $l \leq E_{n, f}:=\operatorname{dist}\left(f, P_{n}\right)$, i.e. for all $f \in C[0,1], n \in \mathbb{N}, l \in \mathbb{Q}_{+}^{*}$ such that $l \leq E_{n, f}$, and $p_{1}, p_{2} \in P_{n}$ :

$$
\bigwedge_{q} \in \mathbb{Q}_{+}^{*}\left(\left\|f-p_{1}\right\|_{\infty},\left\|f-p_{1}\right\|_{\infty} \leq E_{n, f}+(\Phi f n l) \cdot q \rightarrow\left\|p_{1}-p_{2}\right\|_{\infty} \leq q\right) .
$$

Thus -by 2.4.- $\Phi f n l$ (resp. $\frac{2}{\Phi f n l}$ ) is a constant of strong unicity (Lipschitz constant of the Chebcheff projection) of $f, n$ if $E_{n, f} \geq l>0$.

2) $\tilde{\Phi} f n q:=\min \left(\frac{q}{4}, \Phi f n\left(\frac{q}{4}\right) \cdot q\right)$ is a modulus of uniqueness which does not depend on $l$ and the assumption $l \leq E_{n, f}$.

$(n-1:=n-1$, if $n \geq 1$ and $:=0$, if $n=0)$.

The main part of the proof-theoretic analysis yielding 2.7 is the unwinding of the ineffective proof of the alternation theorem which results in a new quantitative version of this theorem: 
Definition 2.8 Suppose $p \in P_{n}, f \in C[0,1], \varepsilon \in \mathbb{R}_{+}$.

1) $\left(x_{1}, \ldots, x_{k}\right) \in[0,1]^{k}(1 \leq k \leq n+2)$ is an $\varepsilon$-alternant of $p-f$ having length $k$ if $x_{1}<\ldots<x_{k}$ and $\bigwedge_{i=1}^{k}\left|(-1)^{i+j}\left(p\left(x_{i}\right)-f\left(x_{i}\right)\right)-E_{n, f}\right| \leq \varepsilon$ for $j=0$ or $j=1$

(Note that for $\varepsilon=0$ and $k=n+2$ this is the usual notion of extremal alternant).

2) $p_{\varepsilon} \in P_{n}$ is an $\varepsilon$-best approximation of $f$ if $\left\|p_{\varepsilon}-f\right\|_{\infty} \leq E_{n, f}+\varepsilon$.

Theorem 2.9 ([21]) Assume that $0 \leq \varepsilon<\frac{E_{n, f}}{4}, 0<q \leq E_{n, f}(\varepsilon, q \in \mathbb{Q})$ and $2 \leq k \leq n+2$. If $p_{\varepsilon} \in P_{n}$ is a

$\prod_{i=1}^{\left\lfloor\frac{k-2}{2}\right\rfloor}\left(2 i-\frac{1}{2}\right) \cdot \prod_{i=1}^{\left\lceil\frac{k-2}{2}\right\rceil}\left(2 i-\frac{3}{2}\right) \cdot \min \left(\frac{1}{n}, \omega_{f, p_{\varepsilon}}\left(\frac{q}{2}\right)\right)^{k-2} \cdot \varepsilon-$ best approximation of $f$, where $\omega_{f, p_{\varepsilon}}$ is a modulus of uniform continuity of $p_{\varepsilon}-f$, then there exists an $\varepsilon$-alternant having length $k$ for $p_{\varepsilon}-f$.

Using the modulus $\tilde{\omega}_{f, n}$ from theorem 2.7 we obtain an estimate for $\varepsilon$-alternation which no longer depends on $p_{\varepsilon}$ :

Corollary 2.10 ([21]) Assume that $0<q \leq E_{n, f}, 0 \leq \varepsilon<E_{n, f}$ and $2 \leq k \leq n+2$. Let $\chi$ be defined by

$$
\chi f n q k:=\left\{\begin{array}{l}
1, \text { if } k=2 \\
\frac{1}{4} \prod_{i=1}^{\left\lfloor\frac{k-2}{2}\right\rfloor}\left(2 i-\frac{1}{2}\right) \cdot \prod_{i=1}^{\left\lceil\frac{k-2}{2}\right\rceil}\left(2 i-\frac{3}{2}\right) \cdot \tilde{\omega}_{f, n}\left(\frac{q}{2}\right)^{k-2}, \text { if } k>2 .
\end{array}\right.
$$

If $p_{\varepsilon} \in P_{n}$ is a $(\chi f n q k) \cdot \varepsilon$-best approximation of $f$ then $p_{\varepsilon}-f$ possesses an $\varepsilon$-alternant of length $k$.

Let us consider now the following question: Is it possible to bypass the complicated analysis of the proof of the alternation theorem (by conceiving this theorem as an analytical assumption of the form $\bigwedge_{x} \in X \bigvee_{y} \in Y_{x}(F(x, y)=0)$ in 2.1-2.3) if one is only interested in a modulus of uniqueness? The alternation theorem has the following logical form:

$$
(*)\left\{\begin{aligned}
& \bigwedge_{p_{b}} \in P_{n}(\| f- p_{b} \|_{\infty}=E_{n, f} \\
& \rightarrow \bigvee_{j} \in\{0,1\},\left(x_{1}, \ldots, x_{n+2}\right) \in[0,1]^{n+2} \\
&\left(\bigwedge_{i=1}^{n+1}\left(x_{i+1}>x_{i}\right) \wedge \bigwedge_{i=1}^{n+2}\left((-1)^{i+j}\left(p\left(x_{i}\right)-f\left(x_{i}\right)\right)=E_{n, f}\right)\right) .
\end{aligned}\right.
$$

For $E_{n, f}>0, \bigwedge_{i=1}^{n+1}\left(x_{i+1}>x_{i}\right)$ is already implied by $\bigwedge_{i=1}^{n+1}\left(x_{i+1} \geq x_{i}\right)$ and the alternation property. Since the alternation theorem is trivial for $E_{n, f}=0$, nothing is lost if we weak $(*)$ to $(* *)$ which results if $\bigwedge_{i=1}^{n+1}\left(x_{i+1}>x_{i}\right)$ is replaced by $\bigwedge_{i=1}^{n+1}\left(x_{i+1} \geq x_{i}\right)$ in $(*)$. (**) has the form

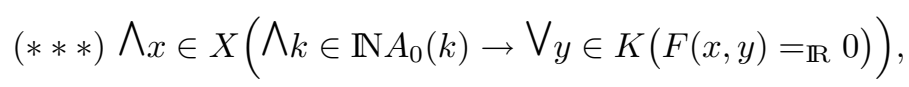

where $A_{0}$ is quantifier-free, $X\left(=C[0,1] \times P_{n}\right)$ is a CSM-space, $K\left(=\{0,1\} \times[0,1]^{n+2}\right)$ is a compact metric space and $F: X \times K \rightarrow \mathbb{R}$ is a constructively definable function. The formula $\bigwedge_{k} \in \mathbb{N} A_{0}(k)$ 
expresses that $\left\|f-p_{b}\right\|_{\infty}=E_{n, f}$. Because of this premise, $(* * *)$ does not have the logical form required in 2.2,2.3 for analytical assumptions $\bigwedge_{x} \in X \bigvee_{y} \in Y_{x}(F(x, y)=0)$ whose proofs don't contribute to the modulus of uniqueness. In [23] it is shown that in general lemmas of the form $(* * *)$ are crucial for the numerical results. There contribution is essentially a modulus $\chi$ such that

$$
\bigwedge_{x} \in X, q \in \mathbb{Q}_{+}^{*}\left(\bigwedge_{k=0}^{\chi x q} A_{0}(k) \rightarrow \bigvee_{y} \in K(|F(x, y)| \leq q)\right) .
$$

It is just such a $\chi$ which is exhibited in 2.10 for the alternation theorem.

However there are other proofs of the uniqueness of the best Chebycheff approximation due to [4] and [32],[29] which use (after some easy modifications) only a consequence of the alternation theorem which has the form $\bigwedge_{x} \in X \bigvee y \in Y_{x}(F(x, y)=0)$ and thus can be conceived simply as an axiom in the course of the proof-theoretic analysis. The unwinding of these proofs, which we carry out now, is much easier (compared to de La Vallée Poussin's proof) and yields significantly better estimates. These estimates are then used to improve results already obtained in $[6],[7],[8]$ substantially.

\section{Proof-theoretic analysis of (a simplification of) the unique- ness proof by Young/Rice}

The proof proceeds as follows: Let $f \in C[0,1],\left(\phi_{1}, \ldots, \phi_{n}\right)$ be a Chebycheff system on $[0,1]$, $H:=\operatorname{Lin}_{\mathbb{R}}\left(\phi_{1}, \ldots, \phi_{n}\right), E_{H, f}:=\operatorname{dist}(f, H)$. Assume that $\psi_{1}, \psi_{2} \in H$ are best approximations of $f$ in $H$, i.e. $\left\|f-\psi_{1}\right\|_{\infty}=E_{H, f}=\left\|f-\psi_{2}\right\|_{\infty}$. By the alternation theorem there exists an alternant $x_{1}<\ldots<x_{n+1}$ in $[0,1]$ for $\psi_{1}-f$, i.e. for $j=0$ or $j=1$ :

$\bigwedge_{i=1}^{n+1}\left((-1)^{i+j}\left(\psi_{1}\left(x_{i}\right)-f\left(x_{i}\right)\right)=E_{H, f}\right)$

Since $\left\|f-\psi_{2}\right\|_{\infty} \leq E_{H, f}$ it follows that $\bigwedge_{i=1}^{n+1}\left((-1)^{i+j}\left(f\left(x_{i}\right)-\psi_{2}\left(x_{i}\right)\right) \geq-E_{H, f}\right)$. Hence

$$
\bigwedge_{i=1}^{n+1}\left((-1)^{i+j}\left(\psi_{1}\left(x_{i}\right)-\psi_{2}\left(x_{i}\right)\right)=(-1)^{i+j}\left(\psi_{1}\left(x_{i}\right)-f\left(x_{i}\right)\right)+(-1)^{i+j}\left(f\left(x_{i}\right)-\psi_{2}\left(x_{i}\right)\right) \geq 0\right) .
$$

Using the fact that $\left(\phi_{1}, \ldots, \phi_{n}\right)$ is a Chebycheff system and $x_{1}<\ldots<x_{n+1}$ one concludes that $\psi_{1} \equiv \psi_{2}$ (This will be shown below). In order to make our results 2.1,2.2,2.3 applicable we restrict $H$ to the compact set $K_{f, H}:=\left\{\psi \in H:\|\psi\|_{\infty} \leq 2\|f\|_{\infty}\right\}$ and modify the proof above in such a way that the alternation theorem is used only in the form

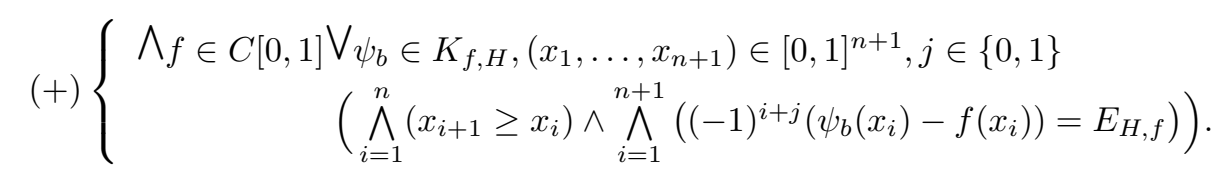

$((+)$ follows immediately from the alternation theorem, the existence of a best approximation of $f$ in $H$ and the fact that each best approximation of $f$ must be in $K_{f, H}$. In the other direction, (+) implies the alternation theorem only when we use already the uniqueness of the best approximation). 
The proof above can be separated into the following parts:

1. $\bigwedge \psi_{1}, \psi_{2} \in K_{f, H},\left(x_{1}, \ldots, x_{n+1}\right) \in[0,1]^{n+1}, j \in\{0,1\}$

$$
\begin{aligned}
\left(\bigwedge _ { i = 1 } ^ { n + 1 } \left((-1)^{i+j}\left(\psi_{1}\left(x_{i}\right)-f\left(x_{i}\right)\right)=\right.\right. & \left.E_{H, f}\right) \wedge \bigwedge_{i=1}^{n+1}\left(\left|\psi_{2}\left(x_{i}\right)-f\left(x_{i}\right)\right| \leq E_{H, f}\right) \\
& \left.\rightarrow \bigwedge_{i=1}^{n+1}\left((-1)^{i+j}\left(\psi_{1}\left(x_{i}\right)-\psi_{2}\left(x_{i}\right)\right) \geq 0\right)\right) .
\end{aligned}
$$

2. $\bigwedge \psi_{1}, \psi_{2} \in K_{f, H},\left(x_{1}, \ldots, x_{n+1}\right) \in[0,1]^{n+1}, j \in\{0,1\}$

$$
\left(\bigwedge_{i=1}^{n}\left(x_{i+1}>x_{i}\right) \wedge \bigwedge_{i=1}^{n+1}\left((-1)^{i+j}\left(\psi_{1}\left(x_{i}\right)-\psi_{2}\left(x_{i}\right)\right) \geq 0\right) \rightarrow\left\|\psi_{1}-\psi_{2}\right\|_{\infty}=0\right) .
$$

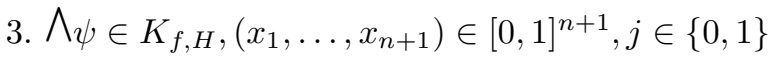

$$
\left(\bigwedge_{i=1}^{n}\left(x_{i+1} \geq x_{i}\right) \wedge \bigwedge_{i=1}^{n+1}\left((-1)^{i+j}\left(\psi\left(x_{i}\right)-f\left(x_{i}\right)\right)=E_{H, f}>0\right) \rightarrow \bigwedge_{i=1}^{n}\left(x_{i+1}>x_{i}\right)\right) .
$$

4. $\bigwedge \psi, \psi_{1}, \psi_{2} \in H\left(\left\|\psi-\psi_{1}\right\|_{\infty}=\left\|\psi-\psi_{2}\right\|_{\infty}=0 \rightarrow\left\|\psi_{1}-\psi_{2}\right\|_{\infty}=0\right)$.

Together with (+), 1.-4. imply that for all $f \in C[0,1]$ :

$$
E_{H, f}>0 \rightarrow \bigwedge \psi_{1}, \psi_{2} \in K_{f, H}\left(\left\|f-\psi_{1}\right\|_{\infty}=E_{H, f}=\left\|f-\psi_{2}\right\|_{\infty} \rightarrow \psi_{1} \equiv \psi_{2}\right) .
$$

Assume $E_{H, f}>0$ : By $(+)$ and 3. there exists $\psi_{b},\left(x_{1}, \ldots, x_{n+1}\right)$ such that (for $\left.j=0\right)$

$$
\bigwedge_{i=1}^{n}\left(x_{i+1}>x_{i}\right) \wedge \bigwedge_{i=1}^{n+1}\left((-1)^{i}\left(\psi_{b}\left(x_{i}\right)-f\left(x_{i}\right)\right)=E_{H, f}>0\right)
$$

By applying 1. to $\psi_{1}^{\prime}:=\psi_{b}, \psi_{2}^{\prime}:=\psi_{1}$ as well as to $\psi_{1}^{\prime}:=\psi_{b}, \psi_{2}^{\prime}:=\psi_{2}$ and $\left(x_{1}, \ldots, x_{n+1}\right)$, it follows that $\bigwedge_{i=1}^{n+1}(-1)^{i}\left(\psi_{b}\left(x_{i}\right)-\psi_{1 / 2}\left(x_{i}\right)\right) \geq 0$ and thus, by $2 .,\left\|\psi_{b}-\psi_{1}\right\|_{\infty}=0$ and $\left\|\psi_{b}-\psi_{2}\right\|_{\infty}=0$. Hence $\psi_{1} \equiv \psi_{2}$.

As said before, the proof of $(+)$ is not relevant (because of its logical form!) for the extraction of the modulus of uniqueness.

1. -4 . can be logically transformed into:

$$
\begin{aligned}
& 1^{*} . \wedge \psi_{1}, \psi_{2} \in K_{f, H},\left(x_{1}, \ldots, x_{n+1}\right) \in[0,1]^{n+1}, j \in\{0,1\}, q \in \mathbb{Q}_{+}^{*} \bigvee_{r} \in \mathbb{Q}_{+}^{*} \\
&\left(\bigwedge _ { i = 1 } ^ { n + 1 } \left((-1)^{i+j}\left(\psi_{1}\left(x_{i}\right)-f\left(x_{i}\right)\right)\right.\right.\left.=E_{H, f}\right) \wedge \bigwedge_{i=1}^{n+1}\left(\left|\psi_{2}\left(x_{i}\right)-f\left(x_{i}\right)\right| \leq E_{H, f}+r\right) \\
&\left.\rightarrow \bigwedge_{i=1}^{n+1}\left((-1)^{i+j}\left(\psi_{1}\left(x_{i}\right)-\psi_{2}\left(x_{i}\right)\right)>-q\right)\right) .
\end{aligned}
$$


$2^{*} . \bigwedge_{\psi_{1}}, \psi_{2} \in K_{f, H},\left(x_{1}, \ldots, x_{n+1}\right) \in[0,1]^{n+1}, j \in\{0,1\}, q, l \in \mathbb{Q}_{+}^{*} \bigvee_{r} \in \mathbb{Q}_{+}^{*}$

$$
\left(\bigwedge_{i=1}^{n}\left(x_{i+1}-x_{i} \geq l\right) \wedge \bigwedge_{i=1}^{n+1}\left((-1)^{i+j}\left(\psi_{1}\left(x_{i}\right)-\psi_{2}\left(x_{i}\right)\right) \geq-r\right) \rightarrow\left\|\psi_{1}-\psi_{2}\right\|_{\infty}<q\right)
$$

$$
\begin{array}{r}
3^{*} . \wedge_{\psi} \in K_{f, H},\left(x_{1}, \ldots, x_{n+1}\right) \in[0,1]^{n+1}, j \in\{0,1\}, q \in \mathbb{Q}_{+}^{*} \bigvee_{r} \in \mathbb{Q}_{+}^{*} \\
\left(\bigwedge_{i=1}^{n}\left(x_{i+1}-x_{i} \geq 0\right) \wedge \bigwedge_{i=1}^{n+1}\left((-1)^{i+j}\left(\psi\left(x_{i}\right)-f\left(x_{i}\right)\right)=E_{H, f} \geq q\right)\right. \\
\left.\rightarrow \bigwedge_{i=1}^{n}\left(x_{i+1}-x_{i}>r\right)\right) .
\end{array}
$$

The quantitative version of 4 . is trivial:

$$
4^{*} . \wedge \psi, \psi_{1}, \psi_{2} \in H, q \in \mathbb{Q}_{+}^{*}\left(\left\|\psi-\psi_{1}\right\|_{\infty},\left\|\psi-\psi_{2}\right\|_{\infty} \leq \frac{q}{2} \rightarrow\left\|\psi_{1}-\psi_{2}\right\|_{\infty} \leq q\right) .
$$

$1^{*}$. and $3^{*}$. can easily be proved in $\mathcal{A}$. As we will see below $2^{*}$. is provable in $\mathcal{A}+$ intermediate value theorem. The intermediate value theorem has the form $\left.\bigwedge_{x} \in X \bigvee_{y \in K(F(x, y)=\mathbb{R}} 0\right)$ with $K$ compact:

$$
\bigwedge_{f \in C[0,1]} \bigvee_{x_{0} \in[0,1]\left(f(0)<0 \wedge f(1)>0 \rightarrow f\left(x_{0}\right)=0\right)}
$$

define $F(f, x):=\min (f(0), 0) \cdot \max (f(1), 0) \cdot f(x)$. Then

$$
F(f, x)=0 \leftrightarrow(f(0)<0 \wedge f(1)>0 \rightarrow f(x)=0) .
$$

Hence we can apply 2.1 to extract bounds from below for " $\bigvee_{r} \in \mathbb{Q}_{+}^{*}$ " (i.e. " $\bigvee_{r} \geq_{\mathbb{Q}} \Phi f q$ ") which depend only on $f, q$ (resp. $f, q, l)$. These bounds realize in fact " $\bigvee_{r} \in \mathbb{Q}_{+}^{*}$ " since $1^{*} .-3^{*}$. are monotonic in $r$. Thus one can obtain effective operations $\Phi_{1}, \Phi_{2}, \Phi_{3}$ such that $\Phi_{1} f q, \Phi_{2} f l q, \Phi_{3} f q \in$ $\mathbb{Q}_{+}^{*}$ for all $q, l \in \mathbb{Q}_{+}^{*}$ and

$$
\begin{aligned}
1^{* *} . & \bigwedge_{\psi_{1}}, \psi_{2} \in K_{f, H},\left(x_{1}, \ldots, x_{n+1}\right) \in[0,1]^{n+1}, j \in\{0,1\}, q \in \mathbb{Q}_{+}^{*} \\
\left(\bigwedge_{i=1}^{n+1}\left((-1)^{i+j}\left(\psi_{1}\left(x_{i}\right)-f\left(x_{i}\right)\right)=E_{H, f}\right)\right. & \wedge \bigwedge_{i=1}^{n+1}\left(\left|\psi_{2}\left(x_{i}\right)-f\left(x_{i}\right)\right| \leq E_{H, f}+\Phi_{1} f q\right) \\
& \left.\rightarrow \bigwedge_{i=1}^{n+1}\left((-1)^{i+j}\left(\psi_{1}\left(x_{i}\right)-\psi_{2}\left(x_{i}\right)\right)>-q\right)\right) .
\end{aligned}
$$

$2^{* *} . \wedge \psi_{1}, \psi_{2} \in K_{f, H},\left(x_{1}, \ldots, x_{n+1}\right) \in[0,1]^{n+1}, j \in\{0,1\}, q, l \in \mathbb{Q}_{+}^{*}$

$$
\begin{aligned}
\left(\bigwedge_{i=1}^{n}\left(x_{i+1}-x_{i} \geq l\right) \wedge \bigwedge_{i=1}^{n+1}\left((-1)^{i+j}\left(\psi_{1}\left(x_{i}\right)-\psi_{2}\left(x_{i}\right)\right) \geq-\Phi_{2} f l q\right)\right. \\
\left.\rightarrow\left\|\psi_{1}-\psi_{2}\right\|_{\infty}<q\right) .
\end{aligned}
$$

$3^{* *} \bigwedge_{\psi} \in K_{f, H},\left(x_{1}, \ldots, x_{n+1}\right) \in[0,1]^{n+1}, j \in\{0,1\}, q \in \mathbb{Q}_{+}^{*}$

$$
\begin{aligned}
\left(\bigwedge _ { i = 1 } ^ { n } ( x _ { i + 1 } - x _ { i } \geq 0 ) \wedge \bigwedge _ { i = 1 } ^ { n + 1 } \left((-1)^{i+j}(\psi\right.\right. & \left.\left.\left(x_{i}\right)-f\left(x_{i}\right)\right)=E_{H, f} \geq q\right) \\
& \left.\rightarrow \bigwedge_{i=1}^{n}\left(x_{i+1}-x_{i}>\Phi_{3} f q\right)\right) .
\end{aligned}
$$


Define $\Phi f l q:=\Phi_{1} f\left(\Phi_{2} f\left(\Phi_{3} f l\right) \frac{q}{2}\right)$. One easily verifies that for all $f \in C[0,1]$ and $l \in \mathbb{Q}_{+}^{*}$ such that $E_{H, f} \geq l$ :

$$
(++) \wedge \psi_{1}, \psi_{2} \in K_{f, H}, q \in \mathbb{Q}_{+}^{*}\left(\left\|\psi_{1}-f\right\|_{\infty},\left\|\psi_{2}-f\right\|_{\infty} \leq E_{H, f}+\Phi f l q \rightarrow\left\|\psi_{1}-\psi_{2}\right\|_{\infty} \leq q\right) .
$$

Only for the verification of $\Phi$, i.e. the proof of $(++)$, the assumption $(+)$ is used. The construction of $\Phi$ does not use (a proof of) (+).

We are now going to construct $\Phi_{1}, \Phi_{2}, \Phi_{3}$ explicitly (in particular for the case $H:=P_{n}$ ):

Ad $\Phi_{1}$ : The construction of $\Phi_{1}$ is trivial. One easily verifies that $\Phi_{1} f q:=q$ fulfils $1^{* *}$. with $\psi_{1}\left(x_{i}\right)-\psi_{2}\left(x_{i}\right) \geq-q$ instead of $\psi_{1}\left(x_{i}\right)-\psi_{2}\left(x_{i}\right)>-q$ for all $\psi_{1}, \psi_{2} \in H$. Its clear that this is sufficient for $(++)$.

Ad $\Phi_{2}$ : Firstly we show that for all $\psi \in H,\left(x_{1}, \ldots, x_{n+1}\right) \in[0,1]^{n+1}$

$$
(*) \bigwedge_{i=1}^{n}\left(x_{i+1}>x_{i}\right) \wedge \bigwedge_{i=1}^{n+1}\left((-1)^{i} \psi\left(x_{i}\right) \geq 0\right) \rightarrow \psi \equiv 0
$$

which immediately gives 2 .

A zero $x^{*} \in(0,1)$ of $\psi$ is called "simple" if $\psi$ changes its sign in $x^{*}$ and "double" else. Assume now that every $\psi \in H, \psi \not \equiv 0$ has at most $n-1$ zeroes if double zeroes count twice. From $\bigwedge_{i=1}^{n+1}(-1)^{i} \psi\left(x_{i}\right) \geq 0$ it follows that $\psi$ has at least $n$ zeroes in this sense and therefore $\psi \equiv 0$. This argument is due to [32] and presented in detail in [29] (pp. 61-62). In order to prove $(*)$ from this, one has to show that double zeroes in fact count twice. This is done e.g. in [29] (p. 57): To every $\psi \in H$ with $\psi \not \equiv 0$ Rice constructs a $\psi_{\varepsilon} \in H$, with $\psi_{\varepsilon} \not \equiv 0$, which has the same simple zeroes as $\psi$ but two simple zeroes for each double zero $y_{i}$ of $\psi\left(\psi\left(y_{i}\right)\right.$ is disturbed by a sufficiently small $\left.\varepsilon\right)$.

We simplify this proof in that we apply such an $\varepsilon$-disturbance directly to the points $x_{1}, \ldots, x_{n+1}$ in $(*)$ and reduce $(*)$ to

$$
(* *) \wedge \psi \in H,\left(x_{1}, \ldots, x_{n+1}\right) \in[0,1]^{n+1} \neg\left(\bigwedge_{i=1}^{n}\left(x_{i+1}>x_{i}\right) \wedge \bigwedge_{i=1}^{n+1}\left((-1)^{i} \psi\left(x_{i}\right)>0\right)\right),
$$

which follows from the intermediate value theorem and the definition of a Chebycheff system.

Lemma 3.1 For all $\psi \in H,\left(x_{1}, \ldots, x_{n+1}\right) \in[0,1]^{n+1}$ the following holds

$$
\bigwedge_{i=1}^{n}\left(x_{i+1}>x_{i}\right) \wedge \bigwedge_{i=1}^{n+1}\left((-1)^{i} \psi\left(x_{i}\right) \geq 0\right) \rightarrow \psi \equiv 0
$$


Proof: Suppose that $\bigwedge_{i=1}^{n}\left(x_{i+1}>x_{i}\right) \wedge \bigwedge_{i=1}^{n+1}\left((-1)^{i} \psi\left(x_{i}\right) \geq 0\right)$ and $(-1)^{i_{0}} \psi\left(x_{i_{0}}\right)=: \alpha>0$ for an $i_{0} \in\{1, \ldots, n+1\}$. By the definition of Chebycheff system there exists a (uniquely determined) $\chi \in H$ such that $\chi\left(x_{i}\right)=(-1)^{i}$ for $i=1, \ldots, i_{0}-1, i_{0}+1, \ldots, n+1$. Let $\varepsilon>0$ be so small that $\varepsilon \cdot\|\chi\|_{\infty}<\alpha$. Then $\bigwedge_{i=1}^{n+1}\left((-1)^{i}(\psi+\varepsilon \chi)\left(x_{i}\right)>0\right)$. But this is impossible by $(* *)$ (since $\left.\psi+\varepsilon \chi \in H\right)$. Hence $\bigwedge_{i=1}^{n+1}\left((-1)^{i} \psi\left(x_{i}\right)=0\right)$ which implies $\psi \equiv 0$.

$(* *)$ is logically equivalent to a purely universal formula (and hence a-fortiori to a formula having the form $\bigwedge_{x} \in X \bigvee_{y} \in K(F(x, y)=0)$ ). Thus by 2.1 we only have to analyse the proof of the implication $(* *) \rightarrow(3.1)$ which can be carried out in $\mathcal{A}$ (if $\left(\phi_{1}, \ldots, \phi_{n}\right)$ is provable in $\mathcal{A}$ a Chebycheff system). An unwinding of the proof of (**) or a constructivation of the proof of 3.1 which uses only $\varepsilon$-instances of the non-constructive intermediate value theorem is neither necessary nor useful.

In the proof of 3.1 we used the norm $\|\chi\|_{\infty}$ of the interpolation "polynomial" $\chi$. Hence for a quantitative version of 3.1 (i.e. for the construction of a $\Phi_{2}$ satisfying $2^{* *}$.) we have to give an upper estimate for $\|\chi\|_{\infty}$ which depends on $q \in \mathbb{Q}_{+}^{*}$ only, where $\bigwedge_{i=1}^{n}\left(x_{i+1}-x_{i} \geq q\right)$, but not on the points $x_{i}$ themselves:

Lemma 3.2 Let $\underline{\phi}=\left\{\phi_{1}, \ldots, \phi_{n}\right\}$ be a Chebycheff system over $[0,1]$ where $\phi_{1}, \ldots, \phi_{n} \in C[0,1]$ are constructively definable and $\underline{\phi}$ is provable in $\mathcal{A}$ plus (possibly)

$\bigwedge_{x} \in X \bigvee_{y} \in Y_{x}\left(F(x, y)={ }_{\mathbb{R}} 0\right)$-lemmas a Chebycheff system. Then one can extract from such a proof a primitive recursive function $\delta: \mathbb{Q}_{+}^{*} \rightarrow \mathbb{Q}_{+}^{*}$ satisfying

$$
\begin{aligned}
\mathcal{A}^{i}+\bigwedge x \in X, n \in \mathbb{N} \bigvee y \in Y_{x}(|F(x, y)| & \left.\leq 2^{-n}\right) \vdash \\
\bigwedge_{\psi} \in H, x_{1}, \ldots, x_{n} \in[0,1], q, r & \in \mathbb{Q}_{+}^{*}\left(\bigwedge_{i=1}^{n-1}\left(x_{i+1}-x_{i} \geq q\right)\right. \\
& \left.\wedge \bigwedge_{i=1}^{n}\left(\left|\psi\left(x_{i}\right)\right| \leq \delta(q) \cdot r\right) \rightarrow\|\psi\|_{\infty} \leq r\right) .
\end{aligned}
$$

(Here $\left.H:=\operatorname{Lin}_{\mathbb{R}}\left(\phi_{1}, \ldots, \phi_{n}\right)\right)$.

Proof: By the assumption that $\underline{\phi}$ is a Chebycheff system we have (provable in $\mathcal{A}+\bigwedge_{x} \in X \bigvee_{\left.y \in Y_{x}(F(x, y)=0)\right)}$

$$
\bigwedge_{m} \in \mathbb{N},\left(x_{1}, \ldots, x_{n}\right) \in N_{m} \bigvee_{k} \in \mathbb{N}\left(|\operatorname{det}(A(\underline{x}))|>2^{-k}\right),
$$

where $N_{m}:=\left\{\left(x_{1}, \ldots, x_{n}\right) \in[0,1]^{n}: \bigwedge_{i=1}^{n-1}\left(x_{i+1}-x_{i} \geq \min \left(\frac{1}{n}, 2^{-m}\right)\right)\right\}$ and $A(\underline{x})$ is the matrix $\left(\phi_{i}\left(x_{j}\right)\right)_{1 \leq i, j \leq n}$. Since $N_{m}$ is compact we can apply 2.1 to obtain a primitive recursive function $\beta: \mathbb{N} \rightarrow \mathbb{N}$ such that 


$$
\begin{aligned}
\mathcal{A}^{i}+\bigwedge x & \in X, n \in \mathbb{N} \bigvee_{y} \in Y_{x}\left(|F(x, y)| \leq 2^{-n}\right) \vdash \\
& \bigwedge_{m} \in \mathbb{N},\left(x_{1}, \ldots, x_{n}\right) \in N_{m}\left(|\operatorname{det}(A(\underline{x}))|>2^{-\beta(m)}\right) .
\end{aligned}
$$

Using $\beta$ one can compute $\left\|A^{-1}(\underline{x})\right\|$ primitive recursively in $\underline{x}$ and therefore also an $\eta: \mathbb{N} \rightarrow \mathbb{N}$ such that $\eta(m) \geq \sup _{\underline{x} \in N_{m}}\left\|A^{-1}(\underline{x})\right\|$, where the norm is given by $\left\|\left(a_{i j}\right)\right\|:=\max _{i=1, \ldots, n} \sum_{k=1}^{n}\left|a_{i k}\right|$. Let $\psi=c_{1} \phi_{1}+\cdots+c_{n} \phi_{n}, m \in \mathbb{N}$ be given and suppose that $\bigwedge_{i=1}^{n}\left|\psi\left(x_{i}\right)\right| \leq \frac{r}{\eta(m)}$ for an $\underline{x} \in N_{m}$. Then $\left\|\left(c_{1}, \ldots, c_{n}\right)\right\|_{\max } \leq r$. Let $K \in \mathbb{N}$ be such that $K \geq \max _{i=1, \ldots, n}\left\|\phi_{i}\right\|_{\infty} .\left\|\left(c_{1}, \ldots, c_{n}\right)\right\|_{\max } \leq r$ implies that $\left\|c_{1} \phi_{1}+\cdots+c_{n} \phi_{n}\right\|_{\infty} \leq r \cdot n \cdot K$. Hence

$$
\delta(m):=(\eta(m) \cdot n \cdot K+1)^{-1},
$$

satisfies 3.2 for $q=2^{-m}$. $\delta$ can be easily extended to $\mathbb{Q}_{+}^{*}$.

In applications of 3.2 to concrete Chebycheff systems one uses of course known results as e.g. interpolation formulas etc.:

Example 3.3 Let $H_{\underline{\Phi}}:=P_{n}\left(=\operatorname{Lin}_{\mathbb{R}}\left(1, X, \ldots, X^{n}\right)\right)$. The fact that $P_{n}$ is a Chebycheff system is easily proved in $\mathcal{A}$ using the interpolation formula of Laplace:

$$
\text { (1) } p(x)=\sum_{i=1}^{n+1} l_{i}(x) \cdot p\left(x_{i}\right), \text { where } l_{i}(x):=\frac{\prod_{j=1, j \neq i}^{n+1}\left(x-x_{j}\right)}{\prod_{j=1, j \neq i}^{n+1}\left(x_{i}-x_{j}\right)} .
$$

Assume $x_{i+1}-x_{i} \geq q$ for $i=1, \ldots, n$. Then

$$
(2)\left|l_{i}(x)\right| \leq \frac{1}{\prod_{j=1, j \neq i}^{n+1} q \cdot|i-j|} \leq \frac{1}{q^{n} \cdot(i-1) !(n-i+1) !} \text { for all } x \in[0,1] .
$$

For $p \in P_{n}$ such that $\bigwedge_{i=1}^{n+1}\left(\left|p\left(x_{i}\right)\right| \leq r\right),(1)$ and (2) imply

$$
|p(x)| \leq \sum_{i=1}^{n+1}\left|l_{i}(x)\right| \cdot\left|p\left(x_{i}\right)\right| \leq r \cdot \sum_{i=1}^{n+1}\left|l_{i}(x)\right| \leq r \cdot \sum_{i=1}^{n+1} \frac{1}{q^{n}(i-1) !(n-i+1) !}
$$

(This inequality can be found also in [5]). One easily verifies that $(i-1) !(n-i+1) ! \geq\left\lfloor\frac{n}{2}\right\rfloor !\left\lceil\frac{n}{2}\right\rceil !$ for $1 \leq i \leq n+1$. Hence $|p(x)| \leq r \cdot \frac{n+1}{\left[\frac{n}{2} ! ! ! \frac{n}{2}\right\rceil ! q^{n}}$.

Therefore $\delta(q):=\frac{\left\lfloor\frac{n}{2}\right\rfloor !\left[\frac{n}{2}\right\rceil !}{n+1} \cdot q^{n}$ fulfils 3.2 . 
Using $\delta$ from 3.2 we are now able to define $\Phi_{2}$ :

Claim 1: $\Phi_{2} f l q:=\delta(l)^{2} \cdot q$ fulfils $2^{* *}$.

Suppose that $(+) \bigwedge_{i=1}^{n}\left(x_{i+1}-x_{i} \geq l\right) \wedge \bigwedge_{i=1}^{n+1}\left((-1)^{i} \psi\left(x_{i}\right)>-\delta(l)^{2} \cdot q\right)$. We have to show that $\|\psi\|_{\infty} \leq q$ : Assume that $\left|\psi\left(x_{i_{0}}\right)\right|>\delta(l) \cdot q$ for an $i_{0} \in\{1, \ldots, n+1\}$. Then $(-1)^{i_{0}} \psi\left(x_{i_{0}}\right)>\delta(l) \cdot q$ since $($ by $(+))$ $(-1)^{i_{0}} \psi\left(x_{i_{0}}\right)>-\delta(l)^{2} \cdot q \geq-\delta(l) \cdot q$ because $\delta(l) \leq 1(\delta(l)>1$ would yield a contradiction when applied to $\chi$ below).

Define $\psi_{q}:=\psi+\delta(l)^{2} \cdot q \cdot \chi$ where $\chi$ is the interpolation "polynomial" from the proof of 3.1. Then for $i \in\{1, \ldots, n+1\} \backslash\left\{i_{0}\right\}$ :

$$
(-1)^{i} \psi_{q}\left(x_{i}\right)=(-1)^{i} \psi\left(x_{i}\right)+(-1)^{i} \delta(l)^{2} \cdot q \cdot(-1)^{i}=(-1)^{i} \psi\left(x_{i}\right)+\delta(l)^{2} \cdot q \stackrel{(+)}{>} 0
$$

and for $i=i_{0}$

$$
(-1)^{i_{0}} \psi_{q}\left(x_{i_{0}}\right)=(-1)^{i_{0}} \psi\left(x_{i_{0}}\right)+(-1)^{i_{0}} \delta(l)^{2} \cdot q \cdot \chi\left(x_{i_{0}}\right) \geq(-1)^{i_{0}} \psi\left(x_{i_{0}}\right)-\delta(l)^{2} \cdot q \cdot\|\chi\|_{\infty}
$$

By 3.2 and the definition of $\chi$ it follows that $\|\chi\|_{\infty} \leq \frac{1}{\delta(l)}$.

Hence $(-1)^{i_{0}} \psi_{q}\left(x_{i_{0}}\right) \geq(-1)^{i_{0}} \psi\left(x_{i_{0}}\right)-\delta(l) \cdot q>0$. In summary we have $\bigwedge_{i=1}^{n+1}(-1)^{i} \psi_{q}\left(x_{i}\right)>0$ which is impossible in view of $(* *)$. Hence $\bigwedge_{i=1}^{n+1}\left|\psi\left(x_{i}\right)\right| \leq \delta(l) \cdot q$ which in turn yields $\|\psi\|_{\infty} \leq q$ by 3.2 .

By a refinement of the reasoning above we can improve $\Phi_{2}$ :

Claim 2: $\Phi_{2} f l q:=\delta(l) \cdot q$ fulfils $2^{* *}$.

Assume $\bigwedge_{i=1}^{n}\left(x_{i+1}-x_{i} \geq l\right) \wedge \bigwedge_{i=1}^{n+1}\left((-1)^{i} \psi\left(x_{i}\right)>-\delta(l) \cdot q\right)$ and the existence of an $x^{*} \in[0,1]$ such that $\left|\psi\left(x^{*}\right)\right|>q$.

Case 1: $\bigvee_{i_{0}} \in\{1, \ldots, n\}: x^{*} \in\left[x_{i_{0}}, x_{i_{0}+1}\right]$.

$1.1(-1)^{i_{0}} \psi\left(x^{*}\right)>q$. Consider $\tilde{x}_{i}:=\left\{\begin{array}{c}x_{i} \text { if } i \neq i_{0} \\ x^{*} \text { if } i=i_{0} .\end{array}\right.$ Let $\chi \in H$ be such that $\chi\left(\tilde{x}_{i}\right)=(-1)^{i}$ for $i=1, \ldots, i_{0}-1, i_{0}+1, \ldots, n+1$ and $\psi_{q}:=\psi+\delta(l) \cdot q \cdot \chi$. Then for $i \in\{1, \ldots, n+1\} \backslash\left\{i_{0}\right\}$

$$
(-1)^{i} \psi_{q}\left(\tilde{x}_{i}\right)=(-1)^{i} \psi_{q}\left(x_{i}\right)=(-1)^{i} \psi\left(x_{i}\right)+(-1)^{i} \delta(l) \cdot q \cdot(-1)^{i}>0
$$

and for $i=i_{0}$

$$
\begin{aligned}
(-1)^{i_{0}} \psi_{q}\left(\tilde{x}_{i_{0}}\right) & =(-1)^{i_{0}} \psi_{q}\left(x^{*}\right)=(-1)^{i_{0}} \psi\left(x^{*}\right)+\delta(l) \cdot q \cdot \chi\left(x^{*}\right) \\
& \geq(-1)^{i_{0}} \psi\left(x^{*}\right)-\delta(l) \cdot q \cdot\|\chi\|_{\infty}>0 \quad\left(\text { since }\|\chi\|_{\infty} \leq \delta(l)^{-1}\right) .
\end{aligned}
$$

In summary we have $\bigwedge_{i=1}^{n+1}(-1)^{i} \psi_{q}\left(\tilde{x}_{i}\right)>0$ which is impossible.

1.2: $(-1)^{i_{0}+1} \psi\left(x^{*}\right)>q$ : Analogous to 1.1 with $i_{0}+1$ instead of $i_{0}$.

The cases $x^{*} \in\left[0, x_{1}\right]$ and $x^{*} \in\left[x_{n+1}, 1\right]$ are treated similarly. Hence $\|\psi\|_{\infty} \leq q$. Altogether we have proved that for $\Phi_{2} f l q:=\delta(l) \cdot q$ :

$$
\begin{aligned}
& \bigwedge \psi_{1}, \psi_{2} \in H,\left(x_{1}, \ldots, x_{n+1}\right) \in[0,1]^{n+1}, j \in\{0,1\}, q, l \in \mathbb{Q}^{*} \\
& \left(\bigwedge_{i=1}^{n}\left(x_{i+1}-x_{i} \geq l\right) \wedge \bigwedge_{i=1}^{n+1}\left((-1)^{i+j}\left(\psi_{1}\left(x_{i}\right)-\psi_{2}\left(x_{i}\right)\right)>-\Phi_{2} f l q\right) \rightarrow\left\|\psi_{1}-\psi_{2}\right\|_{\infty} \leq q\right) .
\end{aligned}
$$


Clearly this also holds if " $>$ " in the premise is weakened to " $\geq$ " (Our conclusion $\left\|\psi_{1}-\psi_{2}\right\|_{\infty} \leq q$ instead of $\left\|\psi_{1}-\psi_{2}\right\|_{\infty}<q$ in $2^{* *}$ is sufficient for the verification of $\left.\Phi\right)$.

Ad: $\Phi_{3}$ : For the construction of $\Phi_{3}$ we need the following explicit version of the fact that $K_{f, H}$ is compact:

Lemma 3.4 Let $\underline{\phi}:=\left\{\phi_{1}, \ldots, \phi_{n}\right\}$ be as in 3.2 and $M \in \mathbb{Q}_{+}^{*}$. Then one can construct a pimitive recursive common modulus $\omega_{H, M}$ of uniform continuity on $[0,1]$ for all $\psi \in H$ such that $\|\psi\|_{\infty} \leq M$ (provable in $\mathcal{A}^{i}+\bigwedge_{x} \in X, n \in \mathbb{N} \bigvee_{y} \in Y_{x}\left(|F(x, y)| \leq 2^{-n}\right)$ ).

Proof: Let $\underline{x}=\left(x_{1}, \ldots, x_{n}\right)$ be defined by $x_{i}:=\frac{i}{n}$ for $i=1, \ldots, n$ and $K \in \mathbb{N}$ such that $K \geq\left\|A^{-1}(\underline{x})\right\|$ (where $\|\cdot\|$ and $A$ are defined as in the proof of 3.2). Then for $\psi=c_{1} \phi_{1}+\ldots+c_{n} \phi_{n}$ with $\|\psi\|_{\infty} \leq M$ it follows that $\left\|\left(c_{1}, \ldots, c_{n}\right)\right\|_{\max } \leq K \cdot M$, i.e. $\bigwedge_{i=1}^{n}\left(\left|c_{i}\right| \leq K \cdot M\right)$. Let $\omega_{\underline{\phi}}$ be a primitive recursive common modulus of uniform continuity for $\phi_{1}, \ldots, \phi_{n}$ and define $\omega_{H, M}(q):=\omega_{\underline{\phi}}\left(\frac{q}{n \cdot K \cdot M}\right)$ for $q \in \mathbb{Q}_{+}^{*}$. For $\psi=c_{1} \phi_{1}+\ldots+c_{n} \phi_{n}$ such that $\|\psi\|_{\infty} \leq M$, we have

$$
\begin{aligned}
|x-y|<\omega_{H, M}(q) \rightarrow|\psi(x)-\psi(y)| & \leq \sum_{i=1}^{n}\left|c_{i}\right| \cdot\left|\phi_{i}(x)-\phi_{i}(y)\right| \\
& <\frac{q}{n \cdot K \cdot M} \sum_{i=1}^{n}\left|c_{i}\right| \leq q .
\end{aligned}
$$

Corollary 3.5 Let $\underline{\phi}$ be as in 3.4, $f \in C[0,1], \omega_{f}$ a modulus of uniform continuity for $f$ and $M \in \mathbb{Q}_{+}^{*}$ such that $M \geq\|f\|_{\infty}$. Then $\omega_{f, H}(q):=\min \left(\omega_{f}\left(\frac{q}{2}\right), \omega_{H, 2 M}\left(\frac{q}{2}\right)\right)$ is a common modulus of uniform continuity for all $\psi-f$ where $\psi \in K_{f, H}$ (in particular for $\psi_{b}-f$ where $\psi_{b}$ is the best approximation of $f$ in $H)$.

Example 3.6 Let $H:=P_{n}, K_{f, n}:=\left\{p \in P_{n}:\|p\|_{\infty} \leq 2\|f\|_{\infty}\right\}$. A very explicit proof of the compactness of $K_{f, n}$ results from the Markov inequality (due to [24]). We apply this inequality directly for the construction of $\omega_{f, n}$ : Markov's inequality says that $\bigwedge_{p} \in P_{n}\left(\left\|p^{\prime}\right\|_{\infty} \leq 2 n^{2} \cdot\|p\|_{\infty}\right)$ (see e.g. [25], p.65). Hence $\bigwedge_{p} \in K_{f, n}\left(\left\|p^{\prime}\right\|_{\infty} \leq 4 n^{2} \cdot\|f\|_{\infty}\right)$. The mean value theorem yields that every $p \in K_{f, n}$ is Lipschitz continuous on $[0,1]$ with Lipschitz constant $4 n^{2}\|f\|_{\infty}$. Hence for all $M \in \mathbb{Q}_{+}^{*}$ with $M \geq\|f\|_{\infty}$ a common modulus of uniform continuity for all $p \in K_{f, n}$ is given by

$$
\omega_{n}^{M}(q):=\left\{\begin{array}{l}
\frac{q}{4 n^{2} M} \text { if } n \geq 1 \\
1 \text { if } n=0
\end{array}\right.
$$

Thus $\omega_{f, n}^{M}(q):=\min \left(\omega_{n}^{M}\left(\frac{q}{2}\right), \omega_{f}\left(\frac{q}{2}\right)\right)$ is a modulus of uniform continuity for all $p-f$ with $p \in K_{f, n}$ if $M \geq\|f\|_{\infty}$.

Using $\omega_{f, H}$ from 3.5 we are now able to construct $\Phi_{3}$ :

$\bigwedge_{i=1}^{n+1}\left((-1)^{i+j}\left(\psi\left(x_{i}\right)-f\left(x_{i}\right)\right)=E_{n, f} \geq q\right)$ implies 
$\bigwedge_{i=1}^{n}\left(\left|\left(\psi\left(x_{i}\right)-f\left(x_{i}\right)\right)-\left(\psi\left(x_{i+1}\right)-f\left(x_{i+1}\right)\right)\right|=2 E_{H, f} \geq 2 q\right) . \quad$ Using $3.5, \psi \in K_{f, H}$ and $\bigwedge_{i=1}^{n}\left(x_{i+1}-x_{i} \geq 0\right)$ this yields $\bigwedge_{i=1}^{n}\left(x_{i+1}-x_{i} \geq \omega_{f, H}(2 q)\right)$. Hence we can define $\Phi_{3} f q:=\omega_{f, H}(2 q) . \Phi_{3}$ satisfies $3^{* *}$. (with " $\geq \Phi_{3} f q$ " instead of " $>\Phi_{3} f q$ " which is sufficient for $(++)$ ).

We are now ready to combine $\Phi_{1}, \Phi_{2}$ and $\Phi_{3}$ to the modulus of uniqueness $\Phi$ :

$$
\Phi f l q:=\Phi_{1} f\left(\Phi_{2} f\left(\Phi_{3} f l\right) \frac{q}{2}\right)=\frac{1}{2} \delta\left(\omega_{f, H}(2 l)\right) \cdot q,
$$

where $\delta, \omega_{f, H}$ are from 3.2 resp. 3.5 .

The restriction to $\psi_{1}, \psi_{2} \in K_{f, H}$ instead of $\psi_{1}, \psi_{2} \in H$ has been used only for the construction of $\Phi_{3}$. However as the proof of $(++)$ shows, $3^{* *}$. is applied only to the best approximation $\psi_{b} \in K_{f, H}$ which by (+) exists. Hence $\Phi$ is not only a modulus of uniqueness on $K_{f, H}$ but on $H$.

Theorem 3.7 Let $\underline{\phi}:=\left\{\phi_{1}, \ldots, \phi_{n}\right\}$ be a Chebycheff system over $[0,1]$ and $\delta: \mathbb{Q}_{+}^{*} \rightarrow \mathbb{Q}_{+}^{*}$ a function such that for all $\psi \in H, x_{1}, \ldots, x_{n} \in[0,1]$

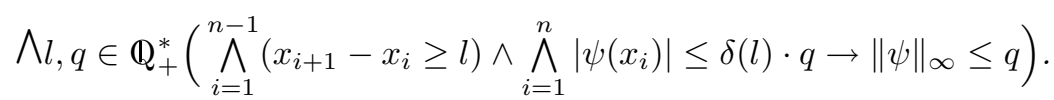

Furthermore let $\omega_{f, H}$ be a common modulus of uniform continuity for all $\psi-f$ with $\psi \in H,\|\psi\|_{\infty} \leq$ $2\|f\|_{\infty}$, and $E_{H, f}:=\operatorname{dist}(f, H)$ where $f \in C[0,1]$. Then the following holds:

1)

$$
\begin{aligned}
& \bigwedge_{f} \in C[0,1], l \in \mathbb{Q}_{+}^{*}\left(l \leq E_{H, f} \rightarrow \bigwedge \psi_{1}, \psi_{2} \in H, q \in \mathbb{Q}_{+}^{*}\right. \\
& \left.\quad\left(\left\|\psi_{1}-f\right\|_{\infty},\left\|\psi_{2}-f\right\|_{\infty} \leq E_{H, f}+\frac{1}{2} \delta\left(\omega_{f, H}(2 l)\right) \cdot q \rightarrow\left\|\psi_{1}-\psi_{2}\right\|_{\infty} \leq q\right)\right),
\end{aligned}
$$

in particular $\delta\left(\omega_{f, H}(2 l)\right)$ is a constant of strong unicity ( and $\frac{2}{\delta\left(\omega_{f, H}(2 l)\right)}$ is a Lipschitz constant for the Chebycheff projection in $f$ ) for $f$ such that $l \leq E_{H, f}$.

2) $\tilde{\Phi} f q:=\min \left(\frac{q}{4}, \frac{1}{2} \delta\left(\omega_{f, H}\left(\frac{q}{2}\right)\right) \cdot q\right)$ is a modulus of uniqueness (and also a modulus of pointwise continuity for the Chebycheff projection) for arbitrary $f \in C[0,1]$ which does not depend on a lower estinate $l \leq E_{H, f}$.

For every constructively definable Chebycheff system $\underline{\phi}$ which is provable in

$\mathcal{A}+\bigwedge_{x} \in X \bigvee_{y} \in Y_{x}\left(F(x, y)={ }_{\mathbb{R}} 0\right)$-lemmas, $\delta$ and $\omega_{f, H}$ are primitive recursively definable and 1),2) above are provable in $\mathcal{A}^{i}+\bigwedge_{x} \in X, n \in \mathbb{N} \bigvee y \in Y_{x}\left(|F(x, y)| \leq 2^{-n}\right)$.

Proof: 1) From the reasoning above it follows that $\frac{1}{2} \delta\left(\omega_{f, H}(2 l)\right) \cdot q$ is a modulus of uniqueness for $f$ such that $l \leq E_{H, f}$. Furthermore if we replace $\psi_{2}$ by the best aproximation $\psi_{b}$ then the factor $\frac{1}{2}$ can be omitted (since it is used only in $4^{*}$.). Hence by $2.4 .2(2.4 .3) \delta\left(\omega_{f, H}(2 l)\right)\left(\right.$ resp. $\left.\frac{2}{\delta\left(\omega_{f, H}(2 l)\right)}\right)$ is a constant of strong unicity (Lipschitz constant).

2) Case 1: $\frac{q}{2}<2 E_{H, f}$ : The claim follows from 1 .

Case $2: \frac{q}{2} \geq 2 E_{H, f}$, i.e. $E_{H, f} \leq \frac{q}{4}$ :

$\left\|\psi_{1}-f\right\|_{\infty},\left\|\psi_{2}-f\right\|_{\infty} \leq E_{H, f}+\tilde{\Phi} f q \leq \frac{q}{4}+\frac{q}{4} \rightarrow\left\|\psi_{1}-\psi_{2}\right\|_{\infty} \leq q$. 
The second part of the claim follows with the proof of 1) and 2.4.1.

$\delta$ and $\omega_{f, H}$ are primitive recursively definable by 3.2 and 3.5 . It remains to show that 1) and 2) are provable in

$\mathcal{A}^{i}+\bigwedge_{x} \in X, n \in \mathbb{N} \bigvee y \in Y_{x}\left(|F(x, y)| \leq 2^{-n}\right)$ : Our analysis of the uniqueness proof uses (besides arguments which are easily provable in $\mathcal{A}$ ) the alternation theorem, the existence of a best approximation and the intermediate value theorem. In [21] it is shown that the alternation theorem is provable in $\mathcal{A}+\mathrm{A}$ where $(\mathrm{A}): \bigwedge_{f} \in C[0,1] \bigvee_{x_{0}} \in[0,1]\left(f\left(x_{0}\right)=\sup _{x \in[0,1]} f(x)\right)$. (A), the existence of a best approximation and the intermediate value theorem are analytical tools having the logical form $\bigwedge_{x} \in \tilde{X} \bigvee \tilde{Y}_{x}(\tilde{F}(x, y)=0)$. Hence (by theorem 2.1) 1) and 2) are provable in $\mathcal{A}^{i}$ plus the $\varepsilon$-weakenings $\bigwedge_{x} \in \tilde{X}, n \in \mathbb{N} \bigvee y \in \tilde{Y}_{x}\left(|\tilde{F}(x, y)| \leq 2^{-n}\right)$ of these tools which are easily proved in $\mathcal{A}^{i}$ (By [31] the intermediate value theorem itself is provable in $\mathcal{A}$, but not in $\mathcal{A}^{i}$. (A) is unprovable in $\mathcal{A}$, but the existence of a best approximation is provable even in $\mathcal{A}^{i}$ by 2.3,2.6).

Using 3.3 and 3.6 we obtain the following example for 3.7 :

Corollary 3.8 Let $\Phi$ be defined by

$$
\text { SfnlM }:=\frac{\left\lfloor\frac{n}{2}\right\rfloor !\left\lceil\frac{n}{2}\right\rceil !}{2(n+1)}\left(\omega_{f, n}^{M}(2 l)\right)^{n}, \text { where } \omega_{f, n}^{M}(q):=\left\{\begin{array}{l}
\min \left(\omega_{f}\left(\frac{q}{2}\right), \frac{q}{8 n^{2} M}\right) \text { if } n \geq 1 \\
1 \quad \text { if } n=0 .
\end{array}\right.
$$

Then

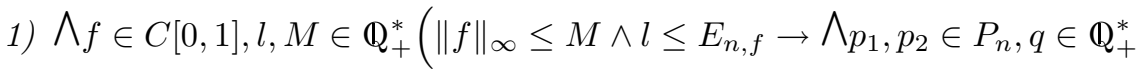

$$
\left.\left(\left\|f-p_{1}\right\|_{\infty},\left\|f-p_{2}\right\|_{\infty} \leq E_{n, f}+(\Phi f n l M) \cdot q \rightarrow\left\|p_{1}-p_{2}\right\|_{\infty} \leq q\right)\right) .
$$

In particular $2 \Phi f n l M$ (resp. $\frac{1}{\Phi f n l M}$ ) is a constant of strong unicity (a Lipschitz constant for the projection) for $f$ if $\|f\|_{\infty} \leq M$ and $l \leq E_{n, f}$.

2) $\tilde{\Phi} f n M q:=\min \left(\frac{q}{4},\left(\Phi f n\left(\frac{q}{4}\right) M\right) q\right)$ is a modulus of uniqueness (and a modulus of pointwise continuity for the projection) for arbitrary $f \in C[0,1]$ with $\|f\|_{\infty} \leq M$.

If $f$ is Lipschitz continuous on $[0,1]$ with Lipschitz constant $\eta>0$ then $\omega_{f, n}^{M}$ can be defined as $\omega_{f, n}^{M}(q):=\frac{q}{\eta+4 n^{2} M}$.

1) and 2) are provable in $\mathcal{A}^{i}$ and thus a-fortiori in constructive analysis in the sense of Bishop.

Remark 3.9 1. Let $A \subset C[0,1]$ be totally bounded with a common modulus of uniform continuity $\omega_{A}$ and $M \geq\|f\|_{\infty}$ for all $f \in A$. Then $\omega_{A, H}(q):=\min \left(\omega_{A}\left(\frac{q}{2}\right), \omega_{H, 2 M}\left(\frac{q}{2}\right)\right)$ (where $\omega_{H, 2 M}$ is from $3.4)$ is a common modulus of uniform continuity for all $\psi-f$ with $f \in A, \psi \in H,\|\psi\|_{\infty} \leq 2\|f\|_{\infty}$. Hence for $0<l \leq \inf _{f \in A} E_{H, f}, 3.7$ yields that $\delta\left(\omega_{A, H}(2 l)\right)\left(\right.$ resp. $\left.\min \left(\frac{q}{4}, \frac{1}{2} \delta\left(\omega_{A, H}\left(\frac{q}{2}\right)\right) \cdot q\right)\right)$ is a common constant of strong unicity (modulus of uniqueness) for all $f \in A$. This is an effective version of a result by [14]: For each compact $A \subset C[0,1]$ such that $A \cap H=\emptyset$, i.e. $\inf _{f \in A} E_{H, f}>0$, there exists a common constant of strong unicity for all $f \in A$.

2. From the proof-analysis above one easily obtains the following result: Let $\left(x_{1}, \ldots, x_{n+1}\right) \in$ 
$[0,1]^{n+1}$ be an alternant of $\psi_{b}-f$ and, for $i=1, \ldots, n+1, \chi_{i}$ the uniquely determined function in $H$ such that $\chi_{i}\left(x_{j}\right)=(-1)^{j}$ for $j \in\{1, \ldots, i-1, i+1, \ldots, n+1\}$. Then $\frac{1}{\max _{i=1, \ldots, n+1}\left\|\chi_{i}\right\|_{\infty}}$ (resp. $\left.2 \cdot \max _{i=1, \ldots, n+1}\left\|\chi_{i}\right\|_{\infty}\right)$ is a constant of strong unicity (Lipschitz constant). This result follows also from Thm. 5 of [11] (using e.g. Thm. 3.5 in [29]), which is proved in a quite different way. Note that these estimates use the knowledge of alternation points which are in general not even recursively computable at all.

3. Our analysis above has similarities with the proof of the Lipschitz continuity of the Chebycheff projection by Freud in [12]. Freud's proof may be conceived as a (partial) proof-analysis in our sense. Although Freud himself does not exhibit the numerical content of his proof one can obtain the estimate of [11] (mentioned in 2.) for the Lipschitz constant quite easily from his proof. This was observed by Blatt in [3] who furthermore noticed that a slight modification of Freud's proof also yields the corresponding estimate for the constant of strong unicity. However neither Freud nor Blatt give a description of constants of strong unicity or Lipschitz constants in which alternation points don't occur (this is in contrast to our results 3.7,3.8 which were obtained before we noticed the papers by Freud and Blatt).

4. The estimates in 3.8 are much better than those obtained from de La Vallée Poussin's proof (2.7): If $\gamma_{Y / R}$ (resp. $\gamma_{P}$ ) denotes the constant of strong unicity obtained from Young/Rice's (resp. de La Vallée Poussin's) proof then $\gamma_{Y / R}$ is roughly $\sqrt[2]{\gamma_{P}}$.

\section{Analysis of a proof from E. Borel:}

We now discuss very briefly a different argument for 3.1 (for the special case $H:=P_{n}$ ) which is used in [4]: Borel gives a proof for the uniqueness of the best polynomial approximation which proceeds as the proof by Young/Rice except that 3.1 for $P_{n}$ can now simply be followed from the fundamental theorem of algebra (This uniqueness proof appeared first in the unpublished part of Kirchberger's dissertation [18]). However in his proof for the continuity of the projection (which easily gives also the Lipschitz continuity) Borel uses a more explicit argument: Using the fact that the derivative $p^{\prime}$ of $p \in P_{n}$ lies in the Haar space $P_{n-1}$ he proves:

$$
\begin{array}{r}
\bigwedge_{p} \in P_{n},\left(x_{1}, \ldots, x_{n+2}\right) \in[0,1]^{n+2}, q \in \mathbb{Q}_{+}^{*}\left(\bigwedge_{i=1}^{n+1}\left(x_{i+1}>x_{i}\right) \wedge \bigwedge_{i=1}^{n+2}\left((-1)^{i} p\left(x_{i}\right)>-q\right)\right. \\
\left.\rightarrow \bigvee_{k} \in\{1, \ldots, n+1\} \bigwedge_{x} \in\left[x_{k}, x_{k+1}\right](|p(x)| \leq q)\right) .
\end{array}
$$

For $q \rightarrow 0$ this gives 3.1, since $\left.p\right|_{\left[x_{k}, x_{k+1}\right]} \equiv 0$ implies $p \equiv 0$. The formula above has the logical form $\bigwedge_{x} \in X \bigvee_{y \in K} \bigwedge_{w} \in W(F(x, y, w)=0)$ and thus it must be possible to extract $\Phi_{2}$ (satisfying $2^{* *}$.) without using the proof of it. In fact this can be done:

$$
x_{k+1}-x_{k} \geq l \rightarrow \bigvee_{y_{1}}, \ldots, y_{n+1} \in\left[x_{k}, x_{k+1}\right]\left(y_{i+1}-y_{i} \geq \frac{l}{n}\right) \text { for } i=1, \ldots, n+1
$$

If this is combined with 3.3 one obtains that

$$
\Phi_{2} f n l q:=\frac{\left\lfloor\frac{n}{2}\right\rfloor !\left\lceil\frac{n}{2}\right\rceil !}{n+1}\left(\frac{l}{n}\right)^{n} \cdot q=\frac{\left\lfloor\frac{n}{2}\right\rfloor !\left\lceil\frac{n}{2}\right\rceil !}{n^{n} \cdot(n+1)} l^{n} \cdot q
$$

fulfils $2^{* *}$. Because of the factor $\frac{1}{n^{n}}$ this gives less good estimates compared to the estimates obtained from our simplification of Young/Rice's proof (specialized to $P_{n}$ ), but better ones than those from 
de La Vallée Poussin's proof (2.7).

Finally we show how 3.7 and 3.9.1 can be used to improve numerical results by D. Bridges in $[6],[7],[8]$ substantially:

Let $\phi:=\left\{\phi_{1}, \ldots, \phi_{n}\right\}$ be a Chebycheff system over $[0,1], \underline{\phi}(x):=\left(\phi_{1}(x), \ldots, \phi_{n}(x)\right) \in \mathbb{R}^{n},\|\underline{\phi}\|:=$ $\sup _{x \in[0,1]}\|\underline{\phi}(x)\|_{2}$, where $\|\cdot\|_{2}$ denotes the Euclidean norm on $\mathbb{R}^{n}$.

$\beta, \gamma, \kappa:\left(0, \frac{1}{n}\right] \rightarrow \mathbb{R}_{+}^{*}$ are defined by

$$
\beta(\alpha):=\left\{\begin{array}{l}
\inf _{x \in[0,1]}\left|\phi_{1}(x)\right|, \text { if } n=1 \\
\inf \left\{\left|\operatorname{det}\left(\phi_{j}\left(x_{i}\right)\right)\right|: 0 \leq x_{1}, \ldots, x_{n} \leq 1, \bigwedge_{i=1}^{n-1}\left(x_{i+1}-x_{i} \geq \alpha\right)\right\}, \text { if } n>1
\end{array}\right.
$$

and

$$
\gamma(\alpha):=\min \left(\|\underline{\phi}\|, \frac{\beta(\alpha)}{n^{\frac{1}{2}}(n-1) ! \prod_{i=1}^{n}\left(1+\left\|\phi_{i}\right\|_{\infty}\right)}\right), \kappa(\alpha):=\gamma(\alpha)^{-1} \cdot\|\underline{\phi}\|
$$

for $\alpha \in\left(0, \frac{1}{n}\right]$. Since $\phi$ is a Chebycheff system it follows that $\beta(\alpha)>0 . H:=\operatorname{Lin}_{\mathbb{R}}\left(\phi_{1}, \ldots, \phi_{n}\right) ; \omega_{\underline{\phi}}$ denotes a modulus of uniform continuity of $\underline{\phi}$. (Bridges uses a slight variant of our notion of modulus of uniform continuity where " $<$ " is replaced by " $\leq$ ". This has the consequence that in 3.11 we have to assume $l_{H, A}<E_{H, A}$ instead of $l_{H, A} \leq E_{H, A}$.)

Lemma 3.10 (Bridges $([6],[7])) \quad$ 1) Suppose that $A \subset C[0,1]$ is totally bounded, $\omega_{A}$ is a common modulus of uniform continuity for all $f \in A$ and $M>0$ is a common bound $M \geq\|f\|_{\infty}$ for all $f \in A$. Then

$$
\omega_{A, H}(\varepsilon):=\min \left(\omega_{A}\left(\frac{\varepsilon}{2}\right), \omega_{\underline{\phi}}\left(\frac{\varepsilon \cdot \beta\left(\frac{1}{n}\right)}{4 M n^{\frac{3}{2}}(n-1) ! \prod_{i=1}^{n}\left(1+\left\|\phi_{i}\right\|_{\infty}\right)}\right)\right)
$$

is a common modulus of uniform continuity for all $\psi_{b}-f$ where $f \in A$ and $\psi_{b}$ is the best approximation of $f$ in $H$.

2) Assume $0<\alpha \leq \frac{1}{n}$ and $\bigwedge_{i=1}^{n-1}\left(x_{i+1}-x_{i} \geq \alpha\right)\left(x_{1}, \ldots, x_{n} \in[0,1]\right)$ for $n \geq 2$. Then

$$
\bigwedge_{\psi} \in H, \varepsilon>0\left(\bigwedge_{i=1}^{n}\left|\psi\left(x_{i}\right)\right| \leq \frac{\gamma(\alpha)}{n \cdot\|\underline{\phi}\|} \cdot \varepsilon \rightarrow\|\psi\|_{\infty} \leq \varepsilon\right)
$$

Proof: 1) See [7] (Lemma). 2) See [6] (4.3).

From 3.7, 3.9.1 and 3.10 we obtain the following

Corollary 3.11 Let $A, \gamma, \kappa$ be as defined before 3.10 and $E_{H, A}:=\inf _{f \in A} E_{H, f}$. Then 3.7 hold uniformly for all $f \in A$ if $E_{H, f}$ is replaced by $E_{H, A}, \omega_{f, H}$ by $\omega_{A, H}$ and $\delta(\alpha)$ by $\frac{\gamma(\alpha)}{n \cdot\|\underline{ }\| \|}$. In particular

$$
\Phi_{A} \varepsilon:=\min \left(\frac{\varepsilon}{4}, \frac{1}{2} \frac{\gamma\left(\min \left(\frac{1}{n}, \omega_{A, H}\left(\frac{\varepsilon}{2}\right)\right)\right)}{n \cdot\|\underline{\phi}\|} \cdot \varepsilon\right)=\min \left(\frac{\varepsilon}{4}, \frac{\varepsilon}{2 n \kappa\left(\min \left(\frac{1}{n}, \omega_{A, H}\left(\frac{\varepsilon}{2}\right)\right)\right)}\right)
$$


is a common modulus of uniqueness (and a common modulus of continuity for the Chebycheff projection in $f$ ) for all $f \in A$.

For $l_{H, A} \in \mathbb{Q}_{+}^{*}$ such that $l_{H, A}<E_{H, A}$ and $0<\alpha \leq \min \left(\frac{1}{n}, \omega_{A, H}\left(2 \cdot l_{H, A}\right)\right)$ we have $\frac{\gamma(\alpha)}{n \cdot\|\underline{\phi}\|}$ (resp. $2 n \kappa(\alpha)$ ) as a uniform constant of strong unicity (resp. Lipschitz constant) for all $f \in A$.

Bridges [6],[7] obtains the following much weaker results:

$n^{-2}\left(\frac{\gamma(\alpha)}{\|\underline{\|}\|}\right)^{2 n+1}$ resp. $2 n \kappa(\alpha) \cdot\left(\sum_{i=1}^{n+1} \kappa(\alpha)^{n+i-1}-1\right)$ where $0<\alpha \leq \min \left(\frac{1}{n}, \omega_{A, H}\left(l_{H, A}\right)\right)$ and $l_{H, A} \leq$ $E_{H, A}$ for strong unicity and for the Lipschitz constant. His modulus of continuity for Chebycheff projection is

$$
\Omega(\varepsilon):=\min \left(\frac{\varepsilon}{8}, \frac{\varepsilon}{2 n \sigma(\varepsilon) \cdot\left(\sum_{i=1}^{n+1} \sigma(\varepsilon)^{n+i-1}-1\right)}\right),
$$

where $\sigma(\varepsilon):=\kappa\left(\min \left(\frac{1}{n}, \omega_{A, H}\left(\frac{\varepsilon}{4}\right)\right)\right)$. A modulus of uniqueness in our sense is constructed by Bridges in $[8]$ but is even worse than $\Omega$. Note that $\frac{\gamma(\alpha)}{\|\underline{\phi}\|}(\leq 1)$ is very close to 0 in practise (e.g. in the case $P_{n-1}$ Bridges takes $\beta(\alpha)$ to be $\left.\alpha^{n(n-1) / 2}\right)$. Hence our results in 3.11 provide an significant improvement of Bridges' estimates. The bounds in 3.11 give effective moduli if $\beta$ can be estimated by a computable function $\tilde{\beta}: \mathbb{Q}_{+}^{*} \rightarrow \mathbb{Q}_{+}^{*}$ such that $0<\tilde{\beta}(\alpha) \leq \beta(\alpha)$ for all $\alpha \in \mathbb{Q}_{+}^{*}$. If $\phi$ is a Chebycheff system as in 3.2 then such a function $\tilde{\beta}$ can be primitive recursively constructed (see the proof of 3.2 ). In practise it will be better to extract the operation $\delta$ in 3.2 directly from a proof of the Chebycheff system property instead of extracting first $\tilde{\beta}$ and then constructing $\gamma$ above via $\tilde{\beta}$ (e.g. for $H:=P_{n}$ our $\delta$ is roughly $\alpha^{n}$ while $\tilde{\beta}$ in this case is already $\left.\alpha^{(n+1) n / 2}\right)$.

From [19] (proofs of thm.4.1 and lemma 4.2) or [20] (proofs of thm.8.30 and lemma 8.29) one can also exhibit a modulus of uniqueness $\Phi$ for the special case $P_{n}$ namely:

$\Phi f n q:=\frac{1}{8(n+1)^{2}} \cdot \omega_{f, n}\left(\frac{q}{2}\right)^{2 n} \cdot q$, where $\omega_{f, n}$ denotes a modulus of continuity for all $p-f$ where $\|p\|_{\infty} \leq 2\|f\|_{\infty}\left(f \in C[0,1], p \in P_{n}\right)$. Ko uses an argument which is similar to our first, rough analysis of the proof of 3.1 (Claim 1!) (see the proof of lemma 4.2 in [19]). However, instead of $\omega_{f, n}$, Ko erroneously uses simply a modulus $\omega_{n}$ for all $p \in P_{n}$ such that $\|p\|_{\infty} \leq 2\|f\|_{\infty}$. Furthermore his modulus $\omega_{n}(q):=q /\left(4\|f\|_{\infty} \cdot n^{2 n} \cdot n^{2}\right)$ is quite bad compared to our $\omega_{n}(q):=q /\left(4\|f\|_{\infty} \cdot n^{2}\right)$ which we used in defining $\omega_{f, n}$. Continuity of the projection and strong unicity are not considered in [19], $[20]$.

D. Bridges works entirely within the framework of constructive analysis in the sense of Bishop, which makes his proofs very complicated. In contrast to this we obtained our results by logical analysis of given classical proofs and determined those parts of the proofs which are relevant for the numerical content (where the possibility of a constructive proof for our resulting estimates is guaranteed by a logical meta-theorem). In our simplification of the uniqueness proof by Young/Rice only small parts had to be analysed since the other parts are lemmas having the form $\bigwedge_{x \in X} \bigvee_{y \in Y_{x}}\left(\bigwedge_{w \in W}\left(F(x, y, w)={ }_{\mathbb{R}} 0\right)\right.$ which can be conceived as new axioms which do not contribute to the logical extraction process. To transform the given proof into a constructive one is neither necessary nor useful. 


\section{References}

[1] Bishop, E., Foundations of constructive analysis. McGraw-Hill, New-York (1967).

[2] Bishop, E., Mathematics as a numerical language. In: Kino, A., Myhill, J., and Vesley, R.E. (eds.) Intuitionism and Proof Theory: Proceedings of the Summer Conference at Buffalo, New York. North-Holland, Amsterdam, pp. 53-71 (1970).

[3] Blatt, H.-P., Lipschitz continuity and strong unicity in G. Freud's work. J. Approximation Theory 46, pp. 25-31 (1986).

[4] Borel, E., Lecons sur les Fonctions de Variables Réelles. Gauthier-Villars, Paris (1905).

[5] Bridges, D.S., On the foundation of best approximation theory. J. Approximation Theory 28, pp. 273-292 (1980).

[6] Bridges, D.S., A constructive developement of Chebychev approximation theory. J. Approximation Theory 30, pp. 99-120 (1980).

[7] Bridges, D.S., Lipschitz constants and moduli of continuity for the Chebyshev projection. Proc. Amer. Math. Soc. 85, pp. 557-561 (1982).

[8] Bridges, D.S., Recent progress in constructive approximation theory. In: Troelstra, A.S./van Dalen, D. (eds.) The L.E.J. Brouwer Centenary Symposium. North-Holland, Amsterdam, pp. 41-50 (1982).

[9] Chebycheff, P.L., Sur les questions de minima qui se rattachent a la représentation approximative des fonctions. Oeuvres I, pp. 273-378 (1859).

[10] Cheney, E.W., Introduction to approximation theory. McGraw-Hill, New-York (1966).

[11] Cline, A.K., Lipschitz conditions on uniform approximation operators. J. Approximation Theory 8, pp. 160-172 (1973).

[12] Freud, G., Eine Ungleichung für Tschebyscheffsche Approximationspolynome. Acta Scientiarum Math. (Szeged) 19, pp. 162-164 (1958).

[13] Gödel, K., Über eine bisher noch nicht benutzte Erweiterung des finiten Standpunktes. Dialectica 12, pp. 280-287 (1958).

[14] Henry, M.S.-Schmidt, D., Continuity theorems for the product approximation operator. In: Law, A.G.-Sahney, B.N. (eds.), Theory of Approximation with Applications, Academic Press, New-York (1976).

[15] Hilbert, D., Über das Unendliche. Math. Ann. 95, pp. 161-190 (1926).

[16] Kleene, S.C., Introduction to metamathematics. North-Holland, Amsterdam (1952).

[17] Jackson, D., Note on a class of polynomials of approximation. Trans. Amer. Math. Soc. 22, pp. 320-326 (1921).

[18] Kirchberger, P., Über Tschebychefsche Annäherungsmethoden. Dissertation, Göttingen (1902). 
[19] Ko, K.-I., On the computational complexity of best Chebyshev approximation. J. of Complexity 2, pp. 95-120 (1986).

[20] Ko, K.-I., Complexity theory of real functions. Birkhäuser; Boston, Basel, Berlin (1991).

[21] Kohlenbach, U., Effective moduli from ineffective uniqueness proofs. An unwinding of de La Vallée Poussin's proof for Chebycheff approximation. To appear in: Ann. Pure Appl. Logic

[22] Kohlenbach, U., Theorie der majorisierbaren und stetigen Funktionale und ihre Anwendung bei der Extraktion von Schranken aus inkonstruktiven Beweisen: Effektive Eindeutigkeitsmodule bei besten Approximationen aus ineffektiven Eindeutigkeitsbeweisen. Dissertation, Frankfurt/Main, pp. xxii+278 (1990).

[23] Kohlenbach, U., Effective bounds from ineffective proofs in analysis: an application of functional interpretation and majorization. J. Symbolic Logic 57, pp. 1239-1273 (1992).

[24] Markov, A.A., Sur une question posee par Mendeleieff. Izvestia Akademii Nauk SSSR 62, pp. $1-24(1889)$.

[25] Meinardus, G., Approximation von Funktionen und ihre numerische Behandlung. Springer Tracts in Natural Philosophy 4, Berlin (1964).

[26] Natanson, I.P., Konstruktive Funktionentheorie. Akademie-Verlag, Berlin (German translations of the original russian edition 1949).

[27] Newman, D.J.-Shapiro, H.S., Some theorems on Cebysev approximation. Duke Math. J. 30, pp. 673-682 (1963).

[28] La Vallée Poussin, C.J. de, Lecons sur l'Approximation des Fonctions d'une Variable Réelle. Gauthier-Villars, Paris (1919).

[29] Rice, J.R., The approximation of functions, vol.1. Addison-Wesley, Reading, Mass. (1964).

[30] Roulier, J.A.--Taylor, G.D., Uniform approximation by polynomials having bounded coefficients. Abh. Math. Sem. Univ. Hamburg 36, pp. 126-135 (1971).

[31] Simpson, S.G., Reverse mathematics. In: Nerode, A.-Shore, R. (eds.), Proceedings of Symposia in Pure Mathematics 42, AMS, Providence, R.I., pp. 461-471 (1985).

[32] Young, J.W., General theory of approximation by functions involving a given number of arbitrary parameters. Trans. Amer.Math. Soc. 8, pp. 331-344 (1907). 